\title{
Detailed Dendritic Excitatory/Inhibitory Balance through Heterosynaptic Spike-Timing-Dependent Plasticity
}

\author{
Naoki Hiratani and ${ }^{-T o m o k i ~ F u k a i ~}$ \\ Laboratory for Neural Circuit Theory, RIKEN Brain Science Institute, Wako, Saitama, Japan 351-0198
}

The balance between excitatory and inhibitory inputs is a key feature of cortical dynamics. Such a balance is arguably preserved in dendritic branches, yet its underlying mechanism and functional roles remain unknown. In this study, we developed computational models of heterosynaptic spike-timing-dependent plasticity (STDP) to show that the excitatory/inhibitory balance in dendritic branches is robustly achieved through heterosynaptic interactions between excitatory and inhibitory synapses. The model reproduces key features of experimental heterosynaptic STDP well, and provides analytical insights. Furthermore, heterosynaptic STDP explains how the maturation of inhibitory neurons modulates the selectivity of excitatory neurons for binocular matching in the critical period plasticity. The model also provides an alternative explanation for the potential mechanism underlying the somatic detailed balance that is commonly associated with inhibitory STDP. Our results propose heterosynaptic STDP as a critical factor in synaptic organization and the resultant dendritic computation.

Key words: critical period; dendritic computation; heterosynaptic plasticity

\section{Significance Statement}

Recent experimental studies reveal that relative differences in spike timings experienced among neighboring glutamatergic and GABAergic synapses on a dendritic branch significantly influences changes in the efficiency of these synapses. This heterosynaptic form of spike-timing-dependent plasticity (STDP) is potentially important for shaping the synaptic organization and computation of neurons, but its functional role remains elusive. Through computational modeling at the parameter regime where previous experimental results are well reproduced, we show that heterosynaptic plasticity serves to finely balance excitatory and inhibitory inputs on the dendrite. Our results suggest a principle of GABA-driven neural circuit formation.

\section{Introduction}

Activity-dependent synaptic plasticity is essential for learning. Especially, the spike-timing difference between presynaptic and postsynaptic neurons is a crucial factor for synaptic learning ( $\mathrm{Bi}$ and Poo, 1998; Caporale and Dan, 2008). Recent experimental results further reveal that the relative differences in spike timings at neighboring synapses on a dendritic branch have a significant influence on changes in synaptic efficiency at these synapses (Tsukada et al., 2005; Hayama et al., 2013; Paille et al., 2013;

Received Jan. 2, 2017; revised 0ct. 10, 2017; accepted 0ct. 17, 2017.

Author contributions: N.H. and T.F. designed research; N.H. performed research; N.H. analyzed data; N.H. and T.F. wrote the paper.

This work was partly supported by JSPS Doctorial Fellowship DC2 (N.H.), CREST JST (JPMJCR13W1 to T.F.), and KAKENHI No15H04265 and No16H01289 (T.F.). We thank Dr. Laurent Venance for kindly providing the experimental data and Dr. Yukiko Goda for comments on the paper.

The authors declare no competing financial interests.

Correspondence should be addressed to Naoki Hiratani, Laboratory for Neural Circuit Theory, RIKEN Brain Science Institute, 2-1 Hirosawa, Wako, Saitama, Japan 351-0198. E-mail: N.Hiratani@gmail.com.

N. Hiratani's present address: Gatsby Computational Neuroscience Unit, University College London, 25 Howland Street, London, UK W1T 4JG.

DOI:10.1523/JNEUROSCI.0027-17.2017

Copyright $\odot 2017$ the authors $\quad 0270-6474 / 17 / 3712106-17 \$ 15.00 / 0$
Bazelot et al., 2015; Oh et al., 2015). In particular, the timing of GABAergic input exerts a great impact on synaptic plasticity at nearby glutamatergic synapses. Similar phenomena have also been observed in biophysical simulations (Cutsuridis, 2011; Bar-Ilan et al., 2013). This heterosynaptic form of spiketiming-dependent plasticity (h-STDP) is potentially important for synaptic organization on the dendritic tree and the resultant dendritic computation (Mel and Schiller, 2004; Branco et al., 2010). However, the functional role of h-STDP remains elusive, partly due to the lack of a simple analytical model.

In the understanding of homosynaptic STDP, simple mathematical formulations of plasticity have played important roles (Gerstner et al., 1996; Song et al., 2000; Vogels et al., 2011). Motivated by these studies, we constructed a mathematical model of h-STDP based on calcium-based synaptic plasticity models (Shouval et al., 2002; Graupner and Brunel, 2012), and then considered the potential functional merits of the heterosynaptic plasticity. The model reproduces several effects of h-STDP that are observed in the hippocampal area CA1 and the striatum of rodents (Hayama et al., 2013; Paille et al., 2013), and provides analytical insights into underlying mechanisms. The model reveals 
Table 1. Definitions of variables

\begin{tabular}{|c|c|c|}
\hline$u_{i}(t)$ & Membrane potential at spine $i$ & Eq. 1 \\
\hline$c_{i}(t)$ & Calcium concentration at spine $i$ & Eq. 3 \\
\hline$y_{i}(t)$ & Interim synaptic weight & Eq. 4 \\
\hline$w_{i}(t)$ & Synaptic weight of spine $i$ & Eq. 5 \\
\hline$g_{\mathrm{N}}(u)$ & Voltage dependence of NMDA receptor & $g_{\mathrm{N}}\left(u_{i}\right)=\alpha_{\mathrm{N}} u_{i}+\beta_{\mathrm{N}}$ \\
\hline$g_{\mathrm{v}}(u)$ & Voltage dependence of VDCC & $g_{\mathrm{v}}\left(u_{i}\right)=\alpha_{\mathrm{v}} u_{i}$ \\
\hline$x_{i}^{A}(t)$ & Inputs through AMPA receptor & Eq. 2 with $Q=A$ \\
\hline$x_{i}^{\mathrm{N}}(t)$ & Inputs through NMDA receptor & Eq. 2 with $Q=N$ \\
\hline$x_{i}^{\mathrm{BP}}(t)$ & Backpropagation & Eq. 2 with $Q=B P$ \\
\hline$x_{i}^{\mathrm{E}}(t)$ & Excitatory heterosynaptic inputs & Eq. 2 with $Q=E$ \\
\hline$x_{i}^{\prime}(t)$ & Inhibitory heterosynaptic inputs & Eq. 2 with $Q=1$ \\
\hline$u_{b}^{k}(\mathrm{t})$ & Membrane potential at dendritic branch $k$ & $u_{b}^{k}(t) \equiv \sum_{i=1}^{N_{b}^{E}} w_{i}^{k} u_{i}^{k}(t) /\left(w_{o}^{E} N_{b}^{E}\right)$ \\
\hline$u_{\text {soma }}(t)$ & Membrane potential at the soma & $u_{\text {soma }}(t) \equiv \sum_{k=1}^{K} g_{b}\left(u_{b}^{k}(t)\right)$. \\
\hline$g_{b}(u)$ & Dendritic nonlinearity function & $g_{b}(u)= \begin{cases}u & \left(\text { if } u>u_{b}^{o}\right) \\
u_{b}^{o} & \text { (otherwise) }\end{cases}$ \\
\hline
\end{tabular}

that h-STDP causes a temporally precise balance (i.e., the detailed balance) between the timing of excitatory and inhibitory inputs on a dendritic branch, because of the inhibitory inputs that shunt long-term depression (LTD) at neighboring correlated excitatory synapses. This result suggests that, not only are the number and total current of excitatory/inhibitory synapses balanced at a branch (Liu, 2004; Wilson et al., 2007), but that the temporal input structure is also balanced, as observed in the soma (Dorrn et al., 2010; Froemke, 2015). Moreover, by considering dendritic computation, we demonstrate that the detailed balance is beneficial for detecting changes in input activity. The model also reconciles with the critical period plasticity for binocular matching observed in the V1 of mice (B. S. Wang et al., 2010, 2013), and provides an explanation for how GABA maturation modulates the selectivity of excitatory neurons during development.

\section{Materials and Methods}

In this study, we first constructed a model of a dendritic spine, and then based on that model, built models of a dendritic branch and a dendritic tree of a neuron. We also created an analytically tractable model of a spine by reducing the original spine model.

Spine model: dynamics. Let us first consider the membrane dynamics of a dendritic spine. The membrane potential of a spine is mainly driven by activation of AMPA/NMDA receptors by presynaptic inputs, backpropagation of postsynaptic spikes, leaky currents, and current influx/outflux caused by excitatory/inhibitory synaptic inputs at nearby synapses. Hence, we modeled the membrane dynamics of a spine $i$ with the following differential equation:

$$
\begin{aligned}
\frac{d u_{i}(t)}{d t}=-\frac{u_{i}(t)}{\tau_{m}}+\gamma_{A} x_{i}^{A}(t) & +\gamma_{N} g_{N}\left(u_{i}\right) x_{i}^{N}(t)+\gamma_{B P} x_{i}^{B P}(t) \\
& -\gamma_{I} \sum_{j \in \Omega_{i}^{I}} x_{j}^{I}\left(t-d_{I}\right)+\gamma_{E} \sum_{j \in \Omega_{i}^{E}} x_{j}^{E}\left(t-d_{E}\right),
\end{aligned}
$$

where $g_{N}\left(u_{i}\right)=\alpha_{\mathrm{N}} u_{i}+\beta_{\mathrm{N}}$, with $\alpha_{\mathrm{N}}$ and $\beta_{\mathrm{N}}$ being constant coefficients. In the equation, $u_{i}$ is the membrane potential of the spine, and $\tau_{\mathrm{m}}$ is the membrane time constant (for definitions of variables, see Table 1 ). Here, changes in conductance were approximated by current changes. The resting potential was normalized to zero for simplicity. The terms, $x_{i}^{\mathrm{A}}$ and $x_{i}^{\mathrm{N}}$ represent the glutamate concentration at AMPA and NMDA receptors, respectively. The function $g_{\mathrm{N}}\left(u_{i}\right)$ represents the voltage dependence of current influx through the NMDA receptors. This positive feedback is enhanced when additional current is provided through backpropagation. As a result, the model reproduces a large depolarization caused by coincident spikes between presynaptic and postsynaptic neurons. In addition, although the AMPA receptor also shows voltage dependence, here we neglected this dependence, as the relative change around the resting potential is small (Lüscher and Malenka, 2012). $x_{i}^{\mathrm{BP}}$ is the effect of backpropagation from the soma, and the last two terms of the equation represents heterosynaptic current, which is given as the sum of the inhibitory (excitatory) currents $x_{j}^{\mathrm{I}}\left(x_{j}^{\mathrm{E}}\right)$ at nearby synapses. We defined the sets of nearby inhibitory and excitatory synapses as $\Omega_{i}^{\mathrm{I}}$ and $\Omega_{i}^{\mathrm{E}}$, respectively, and their delays were denoted as $d_{\mathrm{I}}$ and $d_{\mathrm{E}}$. The parameter for inhibitory heterosynaptic effect $\gamma_{\mathrm{I}}$ is not weight-dependent, because the inhibitory weight was kept constant throughout the paper. In addition, the parameter for excitatory heterosynaptic effect $\gamma_{\mathrm{E}}$ was approximated as a constant in Figure 2, $E$ and $F$, as the synaptic weights changed only slowly, and was set as zero in the rest of simulations.

Each input $x_{i}^{\mathrm{Q}}(\mathrm{Q}=\mathrm{A}, \mathrm{N}, \mathrm{BP}, \mathrm{I}, \mathrm{E})$ is given as the convoluted spikes:

$$
\frac{d x_{i}^{Q}(t)}{d t}=-\frac{x_{i}^{Q}(t)}{\tau_{Q}}+\sum_{s^{k}} \delta\left(t-s^{k}\right),
$$

where $s^{k}$ represents the spike timing of the $k$ th spike. Although convolution is calculated at the heterosynaptic synapse in the simulation, it does not influence the results, as the exponential decay is linear.

Spine model: plasticity. We next consider the calcium influx to a spine through NMDA receptors and the voltage-dependent calcium channel (VDCC). For a given membrane potential $u_{i}$, the calcium concentration at spine $i$ can be written as follows:

$$
\frac{d c_{i}}{d t}=-\frac{c_{i}}{\tau_{C}}+g_{N}\left(u_{i}\right) x_{i}^{N}(t)+g_{V}\left(u_{i}\right)
$$

where $g_{\mathrm{V}}\left(u_{i}\right)=\alpha_{\mathrm{V}} u_{i}$ represents calcium influx through VDCC, and $g_{\mathrm{N}}\left(u_{i}\right)$ $x_{i}^{\mathrm{N}}(t)$ is the influx from NMDA. Importantly, in this configuration, the hyperpolarization of the membrane potential through heterosynaptic inhibitory inputs suppresses the $\mathrm{Ca}^{2+}$ influx to the spine, because both $g_{\mathrm{V}}\left(u_{i}\right)$ and $g_{\mathrm{N}}\left(u_{i}\right)$ are modeled as monotonically increasing functions of the membrane potential. This is consistent with recent findings indicating that an inhibitory input significantly suppresses $\mathrm{Ca}^{2+}$ transients in both dendrites and spines (Chiu et al., 2013; Marlin and Carter, 2014; Müllner et al., 2015). Note that spine-projecting inhibitory synapses might mediate $\mathrm{Ca}^{2+}$ suppression in long-necked spines (Chiu et al., 2013).

The calcium concentration at the spine is a major indicator of synaptic plasticity, and many studies indicate that a high $\mathrm{Ca}^{2+}$ concentration on a spine typically induces LTP, whereas a low concentration often causes LTD, though the speed of $\mathrm{Ca}^{2+}$ rise is also known to affect the sign of plasticity (Lüscher and Malenka, 2012). Previous modeling studies showed that calcium-based synaptic plasticity models constructed on this principle well replicate various homosynaptic STDP time windows observed in in vitro experiments (Shouval et al., 2002; Graupner and Brunel, 2012). We therefore used this framework for modeling plasticity. In contrast to the previous calcium-based model where a synaptic weight was assumed to be binary (Graupner and Brunel, 2012), here we assumed that a synaptic weight is a continuous variable, and we additionally introduced an interim weight variable to ensure that the learning dynamics of synaptic weights is robust. As shown in Equation 5, the interim weight variable imposes an additional threshold mechanism to prevent minor synaptic modulation from affecting the synaptic weight (Petersen et al., 1998). This interim weight variable represents the approximate concentration of plasticity-related enzymes such as CaMKII or PP1 (Graupner and Brunel, 2007). In the proposed model, the interim weight $y_{i}$ and synaptic weight $w_{i}$ follow:

$$
\begin{aligned}
& \frac{d y_{i}(t)}{d t}=-\frac{y_{i}(t)}{\tau_{y}}+C_{p}\left[c_{i}-\theta_{p}\right]_{+}-C_{d}\left[c_{i}-\theta_{d}\right]_{+}, \\
& \frac{d w_{i}(t)}{d t}=B_{p}\left[y_{i}-y_{t h}\right]_{+}-B_{d}\left[-\left(y_{i}+y_{t h}\right)\right]_{+} .
\end{aligned}
$$

$[X]_{+}$is a sign function that returns 1 if $X \geq 0$, but returns 0 otherwise. In the model, the neural dynamics is defined in such a way that the somatic potential caused by a presynaptic spike linearly depends on its synaptic weight $w_{i}$ (Table 1). Thus, $w_{i}$ reflects the amplitude of EPSP. Note that, in this model setting, as observed in recent experiments (Gambino et al., 

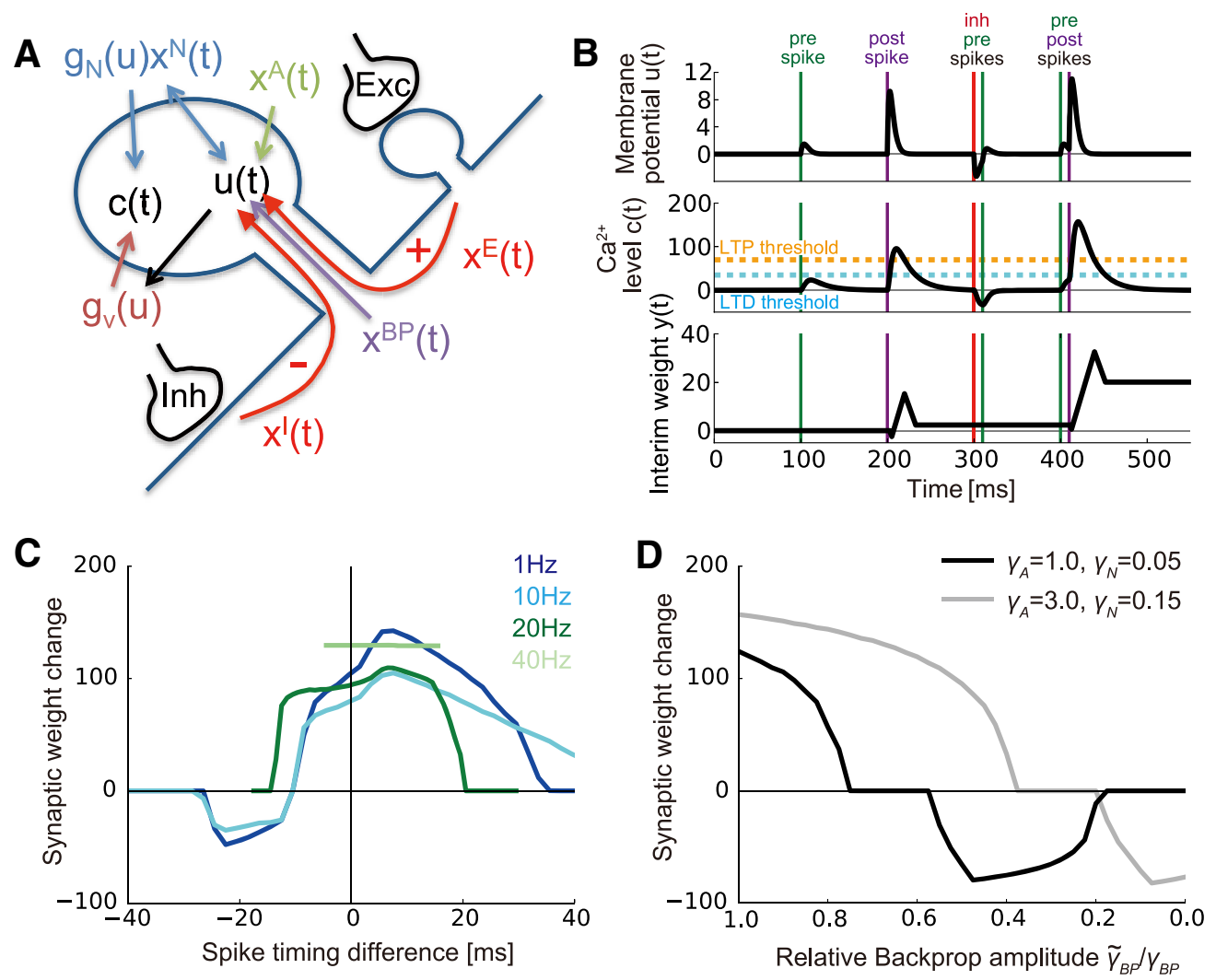

Figure 1. Schematic figure of the model of h-STDP. A, A schematic figure of the model. Two variables in the spine, $u(t)$ and $c(t)$, represent the normalized membrane potential and Ca ${ }^{2+}$ concentration, respectively. Presynaptic action potentials modulate the membrane potential $u(t)$ through $\operatorname{AMPA}\left(x^{\mathrm{A}}\right)$ and $\operatorname{NMDA}\left(g_{\mathrm{N}}(u) x^{\mathrm{N}}\right)$ receptors. In addition, $u(t)$ is modified by backpropagation $\left(x^{\mathrm{BP}}\right)$, and heterosynaptic current caused by excitatory $\left(x^{\mathrm{E}}\right)$ and inhibitory $\left(x^{\mathrm{l}}\right)$ inputs. The calcium level $c(t)$ is modulated by influx/outflux through $\operatorname{NMDA}\left(g_{\mathrm{N}}(u) x^{\mathrm{N}}\right)$ and VDCC $\left[g_{\mathrm{V}}(u)\right]$. Consequently, $c(t)$ is indirectly controlled by $u(t)$, because both NMDA and VDCC are voltage-dependent. $B$, An example of the dynamics of the membrane potential variable $u(t)$ (top), $C a^{2+}$ concentration $c(t)$ (middle), and the interim weight variable $y(t)$ that controls the synaptic weight $w(t)$ (bottom). The change in the $\mathrm{Ca}^{2+}$ level roughly follows the membrane potential dynamics, and the interim weight variable $y(t)$ is positively (negatively) modulated when the $\mathrm{Ca}^{2+}$ level is above the LTP (LTD) thresholds represented by orange (cyan) dotted lines. Based on the variable $y(t)$, the synaptic weight $w(t)$ is updated on a slow timescale (Fig. 5C). C, Pairing frequency dependence of STDP. In corticostriatal synapse model without GABA, we changed the interval of pair-stimulation while keeping the total number of stimulations constant (see Materials and Methods). The blue $1 \mathrm{~Hz}$ line is the same as the red line in Figure $2 A$. The spike-timing windows for 20 and $40 \mathrm{~Hz}$ stimulations were shown only in the middle ranges because the spike-timing difference cannot be longer than the interval of stimulations, and the asymmetry around $0 \mathrm{~ms}$ is caused by the axonal delay. $\boldsymbol{D}$, Dendritic position dependence of STDP. We mimicked dendritic position dependence by changing the amplitude of the backpropagation term $\gamma_{\mathrm{BP}}$ in the corticostriatal synapse model under the pre-post condition depicted by the red lines in Figure $2 B$. In the black line, the AMPA and NMDA coefficients were kept at the same values as in Figure $2 B$, whereas they were increased threefold in the gray line.

2014), backpropagation is not necessary for LTP if presynaptic inputs arrive when the membrane potential at the spine is well depolarized. The model reproduces various properties of homosynaptic STDP replicated by Graupner and Brunel (2012), because it is an extension of their model. For instance, it is known that the STDP time window depends on the frequency of the pre-post stimulation (Sjöström et al., 2001). We confirmed that this could indeed be observed in our model, by changing the interval of stimulation in the simulation of STDP (Fig. 1C). In addition, our model replicates the dendritic position dependence of STDP (Sjöström and Häusser, 2006) by mimicking dendritic attenuation with a reduced backpropagation (Fig. $1 D$, black line). Moreover, by increasing the amplitude of presynaptic stimulation, LTP is rescued (Fig. $1 D$, gray line) as observed in the experiment (Letzkus et al., 2006). Note that Figure 1, $C$ and $D$, corresponds to Graupner and Brunel (2012), their Figures $4 B$ and $5 A$, respectively.

As the heterosynaptic interaction in our model is essentially mediated by the voltage change, our model is also related to the voltage-dependent STDP model (Clopath et al., 2010). The key differences are that our model uses the local membrane potential instead of the somatic potential, and better approximates a previously proposed biophysical model of synaptic plasticity (Graupner and Brunel, 2007) than the phenomenological description used in the voltage-dependent STDP model.

Spine model: details. In the simulation, we set the common parameters as $\tau_{C}=18.0 \mathrm{~ms}, \tau_{\mathrm{M}}=3.0 \mathrm{~ms}, \tau_{\mathrm{N}}=15.0 \mathrm{~ms}, \tau_{\mathrm{A}}=3.0 \mathrm{~ms}, \tau_{\mathrm{BP}}=3.0 \mathrm{~ms}$,
$\tau_{I}=3.0 \mathrm{~ms}, \tau_{\mathrm{E}}=6.0 \mathrm{~ms}, \tau_{\mathrm{Y}}=50 \mathrm{~s}, d_{\mathrm{I}}=0.0 \mathrm{~ms}, \alpha_{\mathrm{N}}=1.0, \beta_{\mathrm{N}}=0.0, \alpha_{\mathrm{V}}=$ 2.0, $\gamma_{\mathrm{A}}=1.0, \theta_{p}=70, \theta_{d}=35, C_{d}=1.0, B_{p}=0.001$, and $B_{d}=0.0005$ (Table 2 shows the definitions and values of the parameters). Note that, due to positive feedback between Equations 1 and 3, the effective timescales of the calcium dynamics and NMDA channels become longer than the given values. In the model of STDP at the striatum, we additionally used $\gamma_{\mathrm{N}}=0.05, \gamma_{\mathrm{BP}}=8.0, \gamma_{\mathrm{I}}=5.0, C_{p}=2.3$, and $y_{\mathrm{th}}=250$, whereas for the model of Schaffer collateral synapses, we used $\gamma_{\mathrm{N}}=0.2, \gamma_{\mathrm{BP}}=8.5, \gamma_{\mathrm{I}}=$ $3.0, C_{p}=2.2, y_{\mathrm{th}}=750, d_{\mathrm{E}}=1.0$, and $\gamma_{\mathrm{E}}=1.0$. In the parameter search, the decay time constants were chosen within biologically reasonable ranges (Koch, 1998); $\alpha_{\mathrm{N}}, \gamma_{\mathrm{A}}, C_{d}$, and $B_{d}$ were fixed at unitary values (i.e., at 1 , except $B_{d}$, which was scaled to 0.0005 ), whereas the other parameters were manually tuned. The robustness of the parameter choices was subsequently confirmed numerically (see Fig. 3). Synaptic weight variables $\{w\}$ were bounded to $0<w<500$, and initialized at $w=w_{\mathrm{o}}^{\mathrm{E}}$, which was defined as $w_{\mathrm{o}}^{\mathrm{E}}=100$. All other variables were initialized at zero in the simulation. Paired stimulation was applied every second for $100 \mathrm{~s}$, and the synaptic weight changes were calculated from the values $400 \mathrm{~s}$ after the end of the stimulation. In the corticostriatal synapse model, the inhibitory spike was presented with the same timing as the presynaptic spike, whereas for the Schaffer collateral synapses, the inhibitory spikes were given $10 \mathrm{~ms}$ before the pre (post) spikes in the pre-post (post-pre) stimulation protocols. In the calculation of the interim weight variable $y(t)$ in Figure 2, $B, D$, and $F$, we ignored the effect of exponential decay 
Table 2. Definitions and values of parameters

\begin{tabular}{|c|c|c|c|c|}
\hline & & $\begin{array}{l}\text { Corticostriatal } \\
\text { model (Fig. 2A,B) }\end{array}$ & $\begin{array}{l}\text { Schaffer-collateral } \\
\text { model (Fig. } 2(-F)\end{array}$ & $\begin{array}{l}\text { Hotspot model, and } \\
\text { single neuron model } \\
\text { (Figs. 5-8) }\end{array}$ \\
\hline$\alpha_{\mathrm{V}}$ & VDCC coefficient & 2.0 & 2.0 & 2.0 \\
\hline$\alpha_{\mathrm{N}}$ & $\begin{array}{l}\text { NMDA voltage } \\
\text { dependence }\end{array}$ & 1.0 & 1.0 & 1.0 \\
\hline$\beta_{\mathrm{N}}$ & NMDA baseline & 0.0 & 0.0 & 1.0 \\
\hline$\gamma_{\mathrm{A}}$ & AMPA coefficient & 1.0 & 1.0 & 1.0 \\
\hline$\gamma_{\mathrm{N}}$ & NMDA coefficient & 0.05 & 0.2 & 0.2 \\
\hline$\gamma_{\mathrm{BP}}$ & $\begin{array}{l}\text { Backpropagation } \\
\text { coefficient }\end{array}$ & 8.0 & 8.5 & 8.0 \\
\hline \multirow[t]{2}{*}{$\gamma_{1}$} & $\begin{array}{l}\text { Inhibitory heterosynaptic } \\
\text { effect }\end{array}$ & 5.0 & 3.0 & 1.2 (Figs. 5-7) \\
\hline & & & & 2.5 (Fig. 8) \\
\hline$\gamma_{\mathrm{E}}$ & $\begin{array}{l}\text { Excitatory heterosynaptic } \\
\text { effect }\end{array}$ & 0.0 & 1.0 & 0.0 \\
\hline$\theta_{p}$ & LTP threshold & 70 & 70 & 70 \\
\hline$\theta_{d}$ & LTD threshold & 35 & 35 & 35 \\
\hline$C_{p}$ & LTP constant & 2.3 & 2.2 & $\begin{array}{l}2.11 \text { in (Figs. 5, 7) } \\
2.01 \text { (Fig. 6) } \\
1.85 \text { (Fig. 8) }\end{array}$ \\
\hline$C_{d}$ & LTD constant & 1.0 & 1.0 & 1.0 \\
\hline$y_{\text {th }}$ & Plasticity threshold & 250 & 750 & $\begin{array}{l}250 \text { (Figs. 5-7) } \\
750 \text { (Fig. 8) }\end{array}$ \\
\hline
\end{tabular}

because of the difference in the timescale $\left(\tau_{y} \gg 1 \mathrm{~s}\right)$. In the calculation of spike-timing difference, we subtracted $7.5 \mathrm{~ms}$ of axonal delay from the timing of presynaptic stimulation. Simulations of the differential equations were implemented using a Runge-Kutta method with a time step of $0.1 \mathrm{~ms}$.

Dendritic hotspot model. A dendritic hotspot model was constructed based on the Schaffer collateral synapse model described above. For simplicity, we hypothesized that the effect of dendritic geometry is negligible within a dendritic hotspot; hence the heterosynaptic current due to the inhibitory spike arrives at all the nearby excitatory spines at the same time. In addition, we also disregarded the excitatory-to-excitatory (Eto-E) interaction by setting $\gamma_{\mathrm{E}}=0.0$. Correlated spikes were generated using hidden variables as in previous studies (Vogels et al., 2011; Hiratani and Fukai, 2015). We generated five dynamic hidden variables and updated them at each time step by $s_{\mu}(t+\Delta t)=\left(\zeta-\frac{1}{2}\right)\left(1-\alpha_{\mathrm{s}}\right)+s_{\mu}(t) \alpha_{s}$, where $\alpha_{s}=\exp \left[-\Delta t / \tau_{S}\right], \tau_{S}=10 \mathrm{~ms}, \mu=0,1, \ldots, 4$, and $\zeta$ is a random variable uniformly chosen from $[0,1)$. In the simulation, the time step was set to $\Delta t=0.1 \mathrm{~ms}$. The activities of the presynaptic neurons were generated by a rate-modulated Poisson process with $r_{i}^{E}(t)=r_{X}^{E}+r_{S}^{E} s_{\mu}(t)$ for excitatory neuron $i$ modulated by the hidden variable $\mu$ (due to a non-negative constraint on $r_{i}^{E}(t)$, we set $r_{i}^{E}(t)=0$ when $r_{X}^{E}+r_{S}^{E} s_{\mu}(t)<0$. Similarly, the presynaptic inhibitory neuron was described by a Poisson model with $r^{I}(t)=r_{X}^{I}+r_{S}^{I} s_{0}(t)$. The activity of the postsynaptic neuron was given as a Poisson model with a fixed rate $r_{\text {post }}$. We set the parameters $\left\{r_{x}^{E}, r_{s}^{E}, r_{\text {post }}\right\}$ such that all presynaptic and postsynaptic excitatory neurons show the same average firing rate at $5 \mathrm{~Hz}$, to avoid the effect of firing-rate differences on synaptic plasticity.

In Figure 7, to explore the role of dendritic spiking in synaptic plasticity, we introduced the effect of dendritic spikes by changing Equation 1 as follows:

$$
\begin{aligned}
\frac{d u_{i}(t)}{d t}=-\frac{u_{i}(t)}{\tau_{m}} & +\gamma_{A} x_{i}^{A}(t)+\gamma_{N} g_{N}\left(u_{i}\right) x_{i}^{N}(t)+\gamma_{B P} x_{i}^{B P}(t) \\
& -\gamma_{I} \sum_{j \in \Omega_{i}^{I}} x_{j}^{I}\left(t-d_{I}\right)+\gamma_{d s}\left[x_{d s}\left(t-d_{E}\right)-\theta_{d s}\right]_{+},
\end{aligned}
$$

where $x_{d s}$ obeys $\frac{d x_{d s}(t)}{d t}=-\frac{x_{d s}(t)}{\tau_{d s}}+\sum_{i} w_{i} \sum_{s_{i}^{k}} \delta\left(t-s_{i}^{k}\right)$, with $s_{i}^{k}$ being the $k$ th spike of the $i$ th presynaptic neuron. The sign function $[X]_{+}$returns 1 if $X \geq 0$, but returns 0 otherwise. With these modifications, the mem- brane potential of each spine receives additional excitatory current when $x_{d s}$ exceeds the threshold $\theta_{d s}$ due to coincident inputs onto nearby spines.

We used $\gamma_{\mathrm{I}}=1.2, \beta_{\mathrm{N}}=1.0, \gamma_{\mathrm{BP}}=8.0, C_{p}=2.11$, and $y_{\text {th }}=250$ with other parameters being kept at the same values used in the original Schaffer collateral model (Table 2). The number of excitatory inputs to the branch $N_{b}^{\mathrm{E}}$ was set to $N_{b}^{\mathrm{E}}=10$. Except for Figure $5 F$, the mean delay of the inhibitory spikes was set to zero. Presynaptic activities were given by $r_{X}^{\mathrm{E}}=$ $1.0 \mathrm{~Hz}, r_{S}^{\mathrm{E}}=500.0, r_{X}^{\mathrm{I}}=2.0 \mathrm{~Hz}$, and $r_{S}^{\mathrm{I}}=1000.0$ so that the average firing rate of presynaptic neurons became $\sim 5 \mathrm{~Hz}$, whereas the postsynaptic firing rate was set to $r_{\text {post }}=5.0 \mathrm{~Hz}$. The large values of $r_{S}^{\mathrm{E}}$ and $r_{S}^{\mathrm{I}}$ were chosen because the correlation factor $s_{\mu}(t)$ was typically very small $\left(\sqrt{\left\langle s_{\mu}^{2}\right\rangle} \approx 0.02\right)$. In Figure $5 C$, the correlations were calculated between the dendritic membrane potential $g_{b}\left(u_{b}\right)$ and hidden variables $\left\{s_{\mu}(t)\right\}$, where $u_{b}(t) \equiv \sum_{i=1}^{N_{b}^{E}} w_{i} u_{i}(t) /\left(w_{o}^{E} N_{b}^{E}\right)$, and $g_{b}(u)$ were defined as $g_{b}(u)=u$ if $u>u_{b}^{\mathrm{o}}$, otherwise $g_{b}(u)=u_{b}^{\mathrm{o}}$ with $u_{b}^{\mathrm{o}}=-5.0$. For the dendritic spike model shown in Figure 7 , we used $\gamma_{\mathrm{sd}}=1.0, \tau_{\mathrm{sd}}=10.0 \mathrm{~ms}$, and $\theta_{\mathrm{ds}}=$ $4 w_{\mathrm{o}}^{\mathrm{E}}$. The other parameters were kept at the same values used above.

Two-layered neuron model. Previous studies suggest that the complicated dendritic computation can be approximated by a two-layered single-cell model (Poirazi et al., 2003; London and Häusser, 2005). We therefore constructed a single cell model by assuming that each hotspot works as a subunit of a two-layered model. In this model, a dendritic subunit (i.e., a unit in the first layer of the two-layered model) corresponds to an electrically compartmentalized subregion of the dendritic tree, such as a thin terminal dendrite or a combination of oblique and terminal branches diverted from the main dendritic shaft. By contrast, in the dendritic hotspot model, a hotspot represents a group of excitatory synapses modulated by a common inhibitory input on a subregion of a dendritic branch. Thus, given that an inhibitory input can modulate excitatory synapses up to $10-15 \mu \mathrm{m}$ away from the input site (Hayama et al., 2013), a dendritic subunit may contain multiple hotspots. However, for simplicity we supposed that a dendritic subunit corresponds to a hotspot in the two-layered model. We defined the mean potential of a dendritic subunit $k$ as $u_{b}^{k}(t) \equiv \sum_{i=1}^{N_{b}^{E}} w_{i}^{k} u_{i}^{k}(t) /\left(w_{o}^{E} N_{b}^{E}\right)$, and calculated the somatic membrane potential as $u_{\text {soma }}(t) \equiv \sum_{k=1}^{K} g_{b}\left(u_{b}^{k}(t)\right)$. Postsynaptic spikes were given as a rate-modulated Poisson model with the rate $u_{\text {soma }}(t) / I_{\mathrm{dv}}(t)$. The term $I_{\mathrm{dv}}(t)$ is a divisive inhibition term introduced to keep the output firing rate at $r_{\text {post }}$. By using the mean somatic potential $\frac{d \bar{u}_{\text {soma }}(t)}{d t}=-\frac{\bar{u}_{\text {soma }}(t)-u_{\text {soma }}(t)}{\tau_{v}}, I_{\mathrm{dv}}(t)$ was calculated as $I_{d v}(t) \equiv \bar{u}_{\text {soma }}(t) / r_{\text {post }}$.

In the simulations described in Figure 6, we used $C_{p}=2.01, \tau_{v}=1 \mathrm{~s}$, and $K=100$, with the other parameters being kept at the same values used in the dendritic hotspot model. During the learning depicted in Figure $6 B-E$, we used the same input configuration as in the dendritic hotspot model. In Figure $6 \mathrm{~F}-H$, the activity level of the hidden variables $\left\{s_{\mu}(t)\right\}$ was kept at a constant value $s_{\mu}(t)=0.25$ during the $500 \mathrm{~ms}$ stimulation, whereas it was otherwise kept at zero. Additionally, the inhibitory presynaptic activities were set to $r_{X}^{\mathrm{I}}=10 \mathrm{~Hz}$ and $r_{S}^{\mathrm{I}}=2000$. For the data presented in Figure 6, $G$ and $H$, we modulated the firing rates of both the excitatory and inhibitory presynaptic neurons by changing the activity levels of the hidden variables $\left\{s_{\mu}(t)\right\}$ from 0.1 to 0.5 . The ratio of the change detecting spikes was defined as the ratio of the spikes occurring within $50 \mathrm{~ms}$ of the change to the total spike count.

In Figure 6, $C$ and $E$, the standard STDP model (Song et al., 2000; Hiratani and Fukai, 2015) was implemented as follows:

$$
\Delta w=\left\{\begin{array}{ll}
\eta_{s t d p} A_{p} \exp \left(-|\Delta t| / \tau_{p}\right) & (\Delta t>0) \\
-\eta_{s t d p} A_{d} \exp \left(-|\Delta t| / \tau_{d}\right) & (\Delta t \leq 0)
\end{array},\right.
$$

where $\Delta t$ is the spike-timing difference between the post and presynaptic spikes (i.e., pre-post is LTP). Here, we introduced a $3 \mathrm{~ms}$ dendritic delay into the calculation of $\Delta t$. In addition, to induce branch-specific competition, we performed normalization $w_{i}=\tilde{w}_{i} / \sum_{i^{\prime} \in \text { branch }}\left(\tilde{w}_{i^{\prime}} / w_{o}\right)$ at each dendritic branch at every time step. Neural dynamics was kept at the same dynamics used in the two-layered model described above. In the simulations presented, we used $\tau_{p}=17 \mathrm{~ms}, \tau_{d}=34 \mathrm{~ms}, A_{p}=1.0, A_{d}=$ 0.5 , and $\eta_{\text {stdp }}=3.0$. 

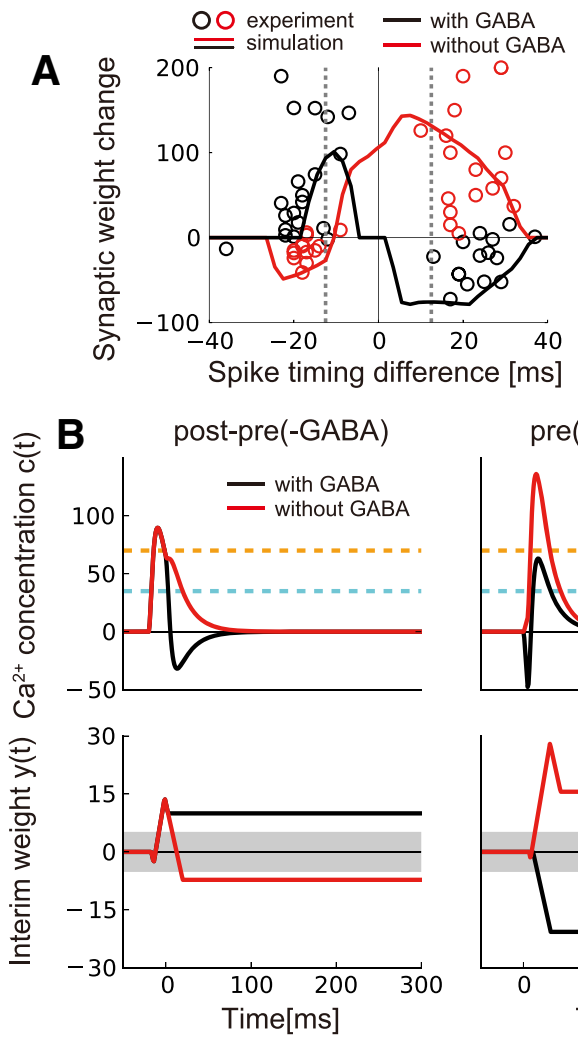

E
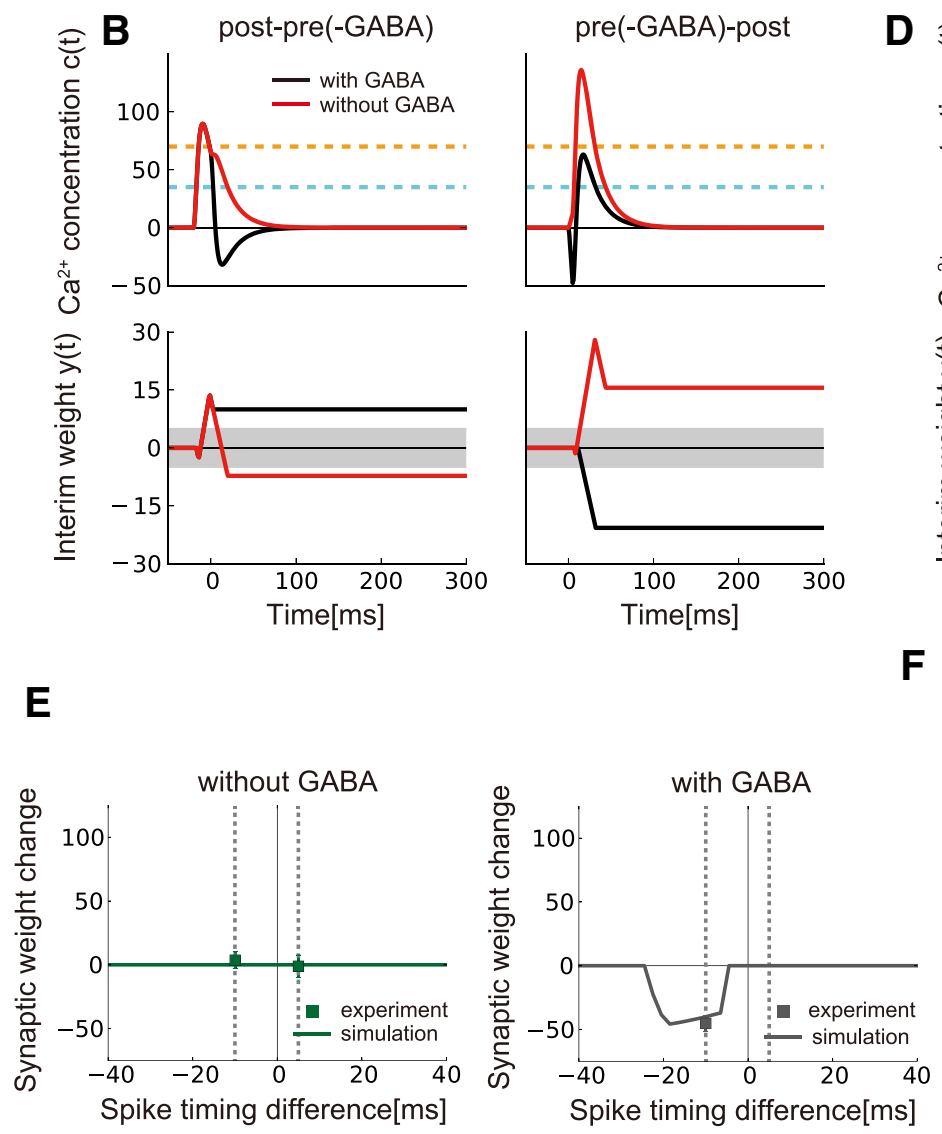

D E

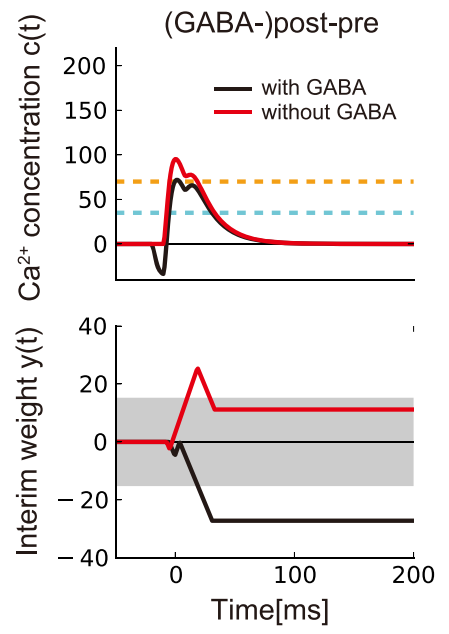

$\mathbf{F}$

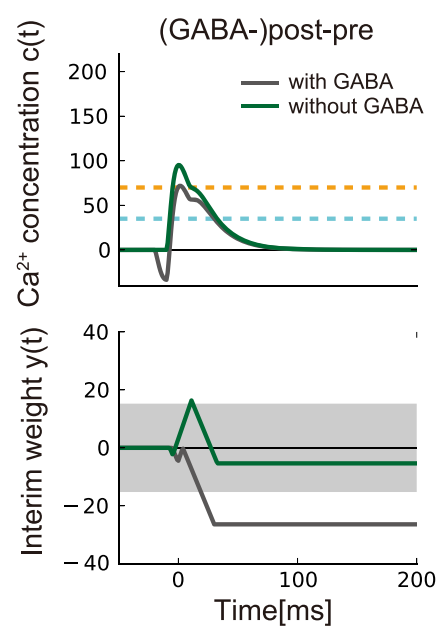

with GABA

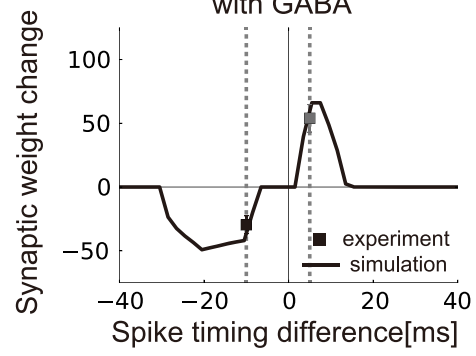

(GABA-)pre-post
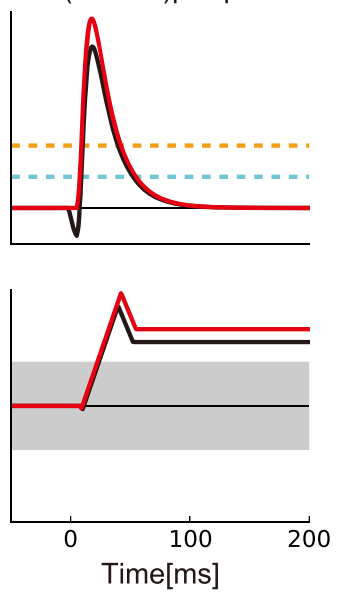

(GABA-)pre-post

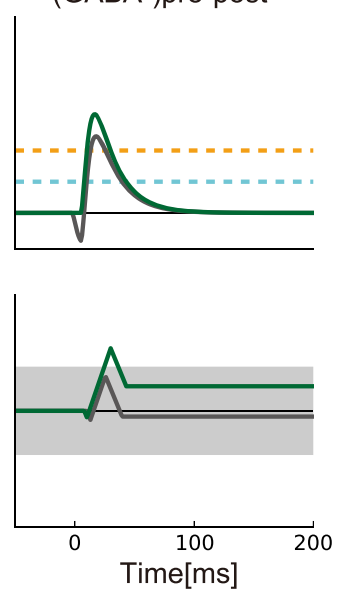

Figure2. The model reproduces spike-timing-dependent heterosynaptic effects. $\boldsymbol{A}$, Spike timing window with/without a disynaptic GABAergic input. The lines represent simulation data, and the points are experimental data taken from Paille et al. (2013). Vertical dotted lines represent the spike-timing differences from which $\boldsymbol{B}$ was calculated. $\boldsymbol{B}$, Dynamics of calcium concentration $(t)$ (top) and the interim weight variable $y(t)$ (bottom) at the stimulated spine. Gray areas in the bottom figures represent the regions satisfying $y(t)<y_{\text {th }} / K_{\text {rep }}$, in which the change in the interim weight is not reflected to the synaptic weight, where $K_{\text {rep }}$ represents the number of paired stimulations given in the simulation for $A$. C, Synaptic weight change with/without GABAergic inputs immediately before pre-post stimulation. Data points were taken from Hayama et al. (2013). The gray point is a result from muscimol application, not GABA uncaging. D, Dynamics of $c(t)$ and $y(t)$ at the stimulated spine. Black lines represent the dynamics under GABA uncaging, and red lines represent the controls. $E$, Synaptic weight change at a neighboring spine through excitatory heterosynaptic interaction. $\boldsymbol{F}$, Dynamics of $c(t)$ and $y(t)$ at the neighboring spine.

The model of binocular matching. For the model of the critical period plasticity of binocular matching depicted in Figure 8, we also used a two-layered single cell model. The neuron model has $K=100$ dendritic branches, each receives $N_{b}^{\mathrm{E}}=20$ excitatory inputs and 1 inhibitory input. At each branch, half of the excitatory inputs are from the contralateral eye, and the other half are from the ipsilateral eye. Each excitatory input neuron has direction selectivity characterized by $\theta_{k, i}^{\mathrm{E}}$, and shows ratemodulated Poisson firing with:

$$
r_{k, i}=r_{x}^{E} \exp \left[\beta_{E} \cos \left(\theta(t)-\theta_{k, i}^{Q}\right)\right] / I_{0}\left(\beta_{E}\right),
$$

where $\theta(t)$ is the direction of the visual stimulus at time $t, Q$ is either contralateral or ipsilateral, and $I_{0}\left(\beta_{\mathrm{E}}\right)$ is the modified Bessel function of order 0 . Similarly, the firing rate of an inhibitory neuron is given as $r_{k}^{I}(t)=r_{x}^{I} \exp \left[\beta_{I} \cos \left(\theta(t)-\theta_{k}^{I}\right)\right] / I_{0}\left(\beta_{I}\right)$. For each excitatory input neuron, the mean direction selectivity $\left\{\theta_{k, i}^{Q}\right\}$ was randomly chosen from a von Mises distribution $\exp \left[\beta_{S} \cos \left(\theta_{k, i}^{Q}-\theta_{Q}\right)\right] / 2 \pi I_{0}\left(\beta_{S}\right)$, where $Q=\{$ contra, ipsi $\}$. In the simulation, we used $\theta_{\text {contra }}=-\pi / 4$, and $\theta_{\text {ipsi }}=\pi / 4$. Correspondingly, the mean direction selectivity of an inhibitory neuron $\left\{\theta_{k}^{I}\right\}$ was defined as the mean of its selectivity for ipsilateral and contralateral inputs (i.e., $\theta_{k}^{I}=\left(\theta_{k}^{I, \text { ipsi }}+\theta_{k}^{\text {I,contra }}\right)$, where $\theta_{k}^{\text {I,ipsi }}$ and $\theta_{k}^{\text {I,contra }}$ were also 
randomly depicted from $\exp \left[\beta_{S} \cos \left(\theta_{k}^{Q}-\theta_{Q}\right)\right] / 2 \pi I_{0}\left(\beta_{S}\right)$. The direction of the visual stimulus $\theta(t)$ changes randomly with $\theta(t+\Delta t)=\theta(t)+\sigma_{s r} \zeta_{G}$, where $\zeta_{G}$ is a Gaussian random variable and $\Delta t$ is the time step of the simulation. To mimic monocular deprivation, in the shadowed area of Figure $8 E$, we replaced the contralateral-driven input neuron activity with a Poisson spiking having a constant firing rate of $r_{m d}^{\mathrm{E}}$. In addition, to simulate the lack of contralateral-driven inputs to inhibitory neurons, we replaced the inhibitory activity with $r_{k}^{I}(t)=r_{m d}^{I}+\left(r_{x}^{I} / 2\right) \exp \left[\beta_{I} \cos \left(\theta(t)-\theta_{k}^{I, i p s i}\right)\right] / I_{0}\left(\beta_{I}\right)$. Similarly, in the firing response shown in Figure $8 C$, we measured direction selectivity by providing monocular inputs, while replacing the inputs from the other eye with homogeneous Poisson spikes with a firing rate of $r_{\mathrm{md}}^{\mathrm{E}}$.

To evaluate the development of binocular matching we introduced three order parameters. First, the difference between the mean excitatory direction selectivity and the inhibitory selectivity at a branch $k$ was evaluated by $\theta_{b, k}^{d}=\left|\arg \left(\sum_{i} w_{k, i}^{E} e^{i\left(\theta_{k, i}^{E}-\theta_{k}^{I}\right)}\right)\right|$. Similarly, the global direction selectivity difference between the inputs from the ipsilateral and contralateral eyes was defined by the following:

$$
\theta_{G}^{d}=\hat{d}\left[\arg \left(\sum_{k=1}^{K} \sum_{i \in \text { ipsi }} w_{k, i}^{E} e^{i \theta_{k, i}^{E}}\right), \arg \left[\sum_{k=1}^{K} \sum_{i \in \text { contra }} w_{k, i}^{E} e^{i \theta_{k, i}^{E}}\right)\right],
$$

where the function $\hat{d}\left[\theta_{1}, \theta_{2}\right]$ calculates the phase difference between the two angles. Finally, the direction selectivity index (DSI) for binocular input was calculated by:

$$
D S I=\left|\sum_{k=1}^{K} \sum_{i=1}^{N_{b}^{E}} w_{k, i}^{E} e^{i \theta_{k, i}^{E}} / \sum_{k=1}^{K} \sum_{i=1}^{N_{b}^{E}} w_{k, i}^{E}\right|
$$

For the calculation of the monocular DSI, at each branch $k$, we took the sum over $N_{b}^{\mathrm{E}} / 2$ excitatory inputs corresponding to each eye, instead of all the $N_{b}^{\mathrm{E}}$ inputs.

In the simulation, we set $\gamma_{\mathrm{I}}=2.5, C_{p}=1.85, y_{\text {th }}=750.0$, and $u_{b}^{\mathrm{o}}=0.0$, with the rest of parameters being kept at the values used in the dendritic hotspot model. The inputs parameters were set to $\beta_{\mathrm{E}}=4.0, \beta_{\mathrm{I}}=2.0$, $\beta_{\mathrm{S}}=1.0, \theta_{\text {contra }}=-\pi / 4, \theta_{\text {ipsi }}=\pi / 4, r_{X}^{\mathrm{E}}=5.0, r_{X}^{\mathrm{I}}=10.0, r_{m d}^{\mathrm{E}}=1.0, r_{m d}^{\mathrm{I}}=$ 1.0 , and $\sigma_{s r}=0.1 \sqrt{\Delta t}$.

Reduced model. If we shrink equations for membrane potential (Eq. 1) and calcium concentration (Eq. 3 ) into one, the reduced equation would be written as follows:

$$
\begin{aligned}
\frac{d C_{i}(t)}{d t}=-\frac{C_{i}(t)}{\tau_{C}}+ & C_{p r e} X_{i}(t)+C_{p o s t}\left[1+g_{C}\left(C_{i}(t-\Delta t)\right)\right] X_{p o s t}(t) \\
& -C_{I} \sum_{j \in \Omega_{i}^{I}} X_{j}^{I}\left(t-d_{I}\right)+C_{E} \sum_{j \in \Omega_{i}^{E}} X_{j}^{E}\left(t-d_{E}\right),
\end{aligned}
$$

where $g_{c}(X)=[X]_{+} \eta X$ captures the nonlinear effect caused by the prepost coincidence [i.e., $g_{c}(X)$ returns $\eta X$ if $X>0$, otherwise returns 0 ]. All inputs $X_{i}, X_{\text {post }}, X_{j}^{\mathrm{I}}$, and $X_{j}^{\mathrm{E}}$ were given as point processes, and $d_{\mathrm{I}}$ and $d_{\mathrm{E}}$ are heterosynaptic delays. The variable $g_{c}$ was calculated from the value of $C_{i}$ at $t=t-\Delta t$ to avoid pathological divergence due to the point processes. In the simulation, we simply used the value of $C_{i}$ from the previous time step. For the interim weight $y$, we used the same equation as before. Note that Equation 11 is basically the same as the one by Graupner and Brunel (2012), except for the nonlinear term $g_{c}(C)$ and the heterosynaptic terms.

Let us consider the weight dynamics of an excitatory synapse that has only one inhibitory synapse in its neighbor. For analytical tractability, we consider the case when presynaptic, postsynaptic, and inhibitory neurons fire only one spikes at $t=t_{\text {pre }}, t_{\text {post }}$, and $t_{\mathrm{I}}$, respectively. In the case of the CA1 experiment, because the GABA uncaging was always performed before the presynaptic and postsynaptic spike, the timing of the inhibitory spike is given as $t_{\mathrm{I}}=\min \left(t_{\text {pre }}, t_{\text {post }}\right)-\delta_{\mathrm{I}}$ for $\delta_{\mathrm{I}}>0$. In this setting, the change in the interim weight variable of the excitatory synapse is given as follows:

$$
\Delta y=\left\{\begin{array}{cc}
G_{1}\left(C_{1}, t_{\text {pre }}-t_{\text {post }}\right) & \left(\text { if } t_{\text {post }}<t_{\text {pre }}\right) \\
+G_{2}\left(C_{\text {pre }}+C_{1} e^{-\left(t_{p r e}-t_{\text {post }}\right) / \tau_{C}}\right) & \\
G_{1}\left(C_{2}, t_{\text {post }}-t_{\text {pre }}\right) & \\
+G_{2}\left(\begin{array}{c}
C_{\text {post }}\left[1+g_{C}\left(C_{2} e^{-\left(t_{\text {post }}-t_{\text {pre }}\right) / \tau_{C}}\right)\right] \\
+C_{2} e^{-\left(t_{\text {post }}-t_{\text {pre }}\right) / \tau_{C}}
\end{array}\right) & \text { (otherwise) }
\end{array},\right.
$$

where

$$
\begin{gathered}
C_{1} \equiv C_{p o s t}-C_{I} e^{-\left(t_{p o s t}-t_{I}\right) / \tau_{C}}, C_{2} \equiv C_{p r e}-C_{I} e^{-\left(t_{p r e}-t_{I}\right) / \tau_{C}}, \\
G_{1}(C, \Delta t) \equiv B_{p}\left[C-\theta_{p}\right]_{+}\left(\left[\tau_{C} \log \frac{C}{\theta_{p}}-\Delta t\right]_{+}\right. \\
\left.\Delta t+\left[\Delta t-\tau_{C} \log \frac{C}{\theta_{p}}\right]_{+} \tau_{C} \log \frac{C}{\theta_{p}}\right)-B_{d}\left[C-\theta_{d}\right]_{+}\left(\left[\tau_{C} \log \frac{C}{\theta_{d}}-\Delta t\right]_{+}\right. \\
\left.\Delta t+\left[\Delta t-\tau_{C} \log \frac{C}{\theta_{d}}\right]_{+} \tau_{C} \log \frac{C}{\theta_{d}}\right), \\
G_{2}(C) \equiv B_{p}\left[C-\theta_{p}\right]_{+} \tau_{C} \log \frac{C}{\theta_{p}}-B_{d}\left[C-\theta_{d}\right]_{+} \tau_{C} \log \frac{C}{\theta_{d}} .
\end{gathered}
$$

Similarly, in the case of the striatum experiment, by setting $\eta=0$, the change in the interim weight variable is given as follows:

$$
\begin{aligned}
& \Delta y \\
& =\left\{\begin{array}{cc}
G_{1}\left(C_{\text {post }}, t_{\text {pre }}-t_{\text {post }}\right)+G_{1}\left(C_{3}, t_{I}-t_{\text {pre }}\right) & \left(\text { if } t_{\text {post }}<t_{\text {pre }}<t_{I}\right) \\
+G_{2}\left(-C_{I}+C_{3} e^{-\left(t_{I}-t_{p r e}\right) / \tau_{C}}\right) & \\
G_{1}\left(C_{p r e}, t_{I}-t_{p r e}\right)+G_{1}\left(C_{4}, t_{\text {post }}-t_{I}\right) & \left(\text { if } t_{\text {pre }}<t_{I}<t_{\text {post }}\right) \\
+G_{2}\left(C_{\text {post }}+C_{4} e^{-\left(t_{\text {post }}-t_{I}\right) / \tau_{C}}\right) & \\
G_{1}\left(C_{\text {pre }}, t_{\text {post }}-t_{\text {pre }}\right)+G_{1}\left(C_{5}, t_{I}-t_{\text {post }}\right) & \text { (if } \left.t_{\text {pre }}<t_{\text {post }}<t_{I}\right) \\
+G_{2}\left(-C_{I}+C_{5} e^{-\left(t_{I}-t_{p o s t}\right) / \tau_{C}}\right) &
\end{array}\right.
\end{aligned}
$$

where

$$
\begin{aligned}
C_{3} \equiv C_{p r e}+C_{p o s t} e^{-\left(t_{p r e}-t_{p o s}\right) / \tau_{C}}, C_{4} \equiv-C_{I}+C_{p r e} e^{-\left(t_{I}-t_{p r e}\right) / \tau_{C}} \\
\\
\text { and } C_{5} \equiv C_{p o s t}+C_{p r e} e^{-\left(t_{p o s t}-t_{p r e}\right) / \tau_{C}} .
\end{aligned}
$$

In the simulation, the parameters were set to $\tau_{c}=30 \mathrm{~ms}, C_{\text {post }}=2.0$, $\theta_{p}=1.6, \theta_{d}=1.0, B_{p}=2.25$, and $B_{d}=1.0$. Additionally, in the model of a Schaffer collateral synapse, we used $\delta_{\mathrm{I}}=1.0, C_{\mathrm{pre}}=1.0, C_{\mathrm{E}}=0.30$, and $\eta=2.0$, whereas for the model of a corticostriatal synapse, we used $\delta_{\mathrm{I}}=$ $5.0, C_{\text {pre }}=0.75, C_{\mathrm{E}}=0.0$, and $\eta=0.0$. In Figures $4, C$ and $D$, we used the parameter set for the model of a Schaffer collateral synapse.

As depicted in Figure 4D, the model also provides an analytical insight into the E-to-E interaction, in addition to the inhibitory-to-excitatory (I-to-E) interaction analyzed in the main result. In the E-to-E interaction, neighboring synapses receive small heterosynaptic calcium transient $C_{E}$, instead of presynaptic input $C_{\text {pre. }}$. We can therefore characterize the shapes of the STDP time windows by the heterosynaptic excitatory effect parameter $C_{\mathrm{E}}$, and the postsynaptic effect parameters $C_{\text {post }}$ (Fig. $4 D$ ). When the postsynaptic effect parameter $C_{\text {post }}$ satisfies $\theta_{p}<C_{\text {post }}<\theta_{p}+C_{I} e^{-\delta_{I} / \tau_{C}}$, and the heterosynaptic effect parameter $C_{\mathrm{E}}$ fulfills $C_{I} e^{-\delta_{I} / \tau_{C}}<C_{E}<\theta_{p}$, the STDP time window shows Hebbian-type timing dependency (Fig. $4 D$, top-middle orange region). However, if $C_{\mathrm{E}}$ is smaller than $C_{I} e^{-\delta_{I} / \tau_{C}}$ while satisfying $\theta_{p}+C_{I} e^{-\delta_{I} / \tau_{C}}-C_{\text {post }}<C_{E}$, then the STDP curve becomes LTD dominant (Fig. $4 D$, top-left green region), as observed in previous experiments (Hayama et al., 2013; Oh et al., 2015). The excitatory heterosynaptic effect $C_{\mathrm{E}}$ is expectedly smaller than the inhibitory effect $C_{\mathrm{I}}$, because the inhibitory potential is typically more localized (Gidon and Segev, 2012). Thus, $C_{E}<C_{I} e^{-\delta_{I} / \tau_{C}}$ is also expected to hold for small $\delta_{\mathrm{I}}$, suggesting robust heterosynaptic LTD at neighboring synapses. 
Experimental design and statistical analysis. Parameters used in the simulations are summarized in Table 2. The main simulation codes for the models are available at https://github.com/nhiratani/hstdp.

\section{Results}

Calcium-based synaptic plasticity model with current-based heterosynaptic interaction explains h-STDP

We constructed a model of a dendritic spine, as shown in Figure $1 A$ (see Materials and Methods, Spine model). In the model, the membrane potential of the spine $u(t)$ is modulated by the current influx/outflux via the AMPA and NMDA receptors (Fig. 1A, $x^{\mathrm{A}}$ and $\left.g_{\mathrm{N}}(u) x^{\mathrm{N}}\right)$, spike backpropagation $\left(x^{\mathrm{BP}}\right)$, and heterosynaptic currents from nearby excitatory and inhibitory synapses $\left(x^{\mathrm{E}}\right.$ and $x^{\mathrm{I}}$, respectively; see Table 1 for the definitions of variables). The calcium concentration in the spine $c(t)$ is controlled through the NMDA receptors and the VDCCs $g_{\mathrm{v}}(u)$ (Higley and Sabatini, 2012). Because both NMDA and VDCC are voltage-dependent (Lüscher and Malenka, 2012), the calcium level in the spine is indirectly controlled by presynaptic, postsynaptic, and heterosynaptic activities (Fig. 1B, top and middle). The voltage dependence of NMDA and VDCC $\left[g_{\mathrm{N}}(u)\right.$ and $\left.g_{\mathrm{v}}(u)\right]$ were assumed to be linear for simplicity. This linear assumption may overestimate the effect of a backpropagating action potential on the dendritic NMDA receptors in the overshooting phase, but the effect is expectedly insignificant because in the absence of $\mathrm{Ca}^{2+}$ spike, a backpropagated action potential tends to overshoot less at the dendrite compared with the soma due to dendritic attenuation (London and Häusser, 2005). In addition, although the synaptic input at an inhibitory synapse causes a positive $\mathrm{Ca}^{2+}$ influx by itself (Koch, 1998), activation of nearby inhibitory input hyperpolarizes the membrane potential at the local dendritic site, and may suppress the $\mathrm{Ca}^{2+}$ influx through VDCCs. Indeed, recent experimental results reveal that $\mathrm{Ca}^{2+}$ influx driven by backpropagating spikes or excitatory synaptic inputs is strongly reduced by an inhibitory input in a temporally and spatially precise manner (Hayama et al., 2013; Marlin and Carter, 2014; Müllner et al., 2015). On the basis of these observations, the $\mathrm{Ca}^{2+}$ level in our model is negatively regulated by the heterosynaptic inhibitory inputs through the hyperpolarization of the membrane potential. To model synaptic plasticity, we used a calcium-based model in which LTP/LTD are initiated if the $\mathrm{Ca}^{2+}$ level reaches above the LTP/LTD thresholds (Fig. 1B, middle, orange and cyan lines); this plasticity model reproduces the features of homosynaptic STDP very well (Shouval et al., 2002; Graupner and Brunel, 2012). We introduced the interim weight variable $y(t)$ to capture the non-graded nature of synaptic weight change (Petersen et al., 1998). Thus, changes in $\mathrm{Ca}^{2+}$ level are first embodied in the interim weight $y(t)$ (Fig. $1 B$, bottom), and are then reflected in the synaptic weight $w(t)$ upon accumulation. The interim weight $y(t)$ is expected to correspond with the concentration of active plasticity-related enzymes such as CaMKII or PP1 (Graupner and Brunel, 2007), and the synaptic weight $w(t)$ reflects the somatic EPSP amplitude. Despite the modification, our model replicates the properties of homosynaptic STDP reproduced by Graupner and Brunel (2012) well (Fig. 1C,D).

We first consider the effect of inhibitory input on synaptic plasticity at nearby excitatory spines. A recent experimental study in a medium spiny neuron (Paille et al., 2013) revealed that synaptic connections from cortical excitatory neurons typically show anti-Hebbian type STDP under a pairwise stimulation protocol, but if the GABA-A receptor is blocked, the STDP time window flips to a Hebbian type STDP (Fig. $2 A$, circles). Our model can explain this phenomenon in the following way. Let us first con- sider the case when the presynaptic excitatory input arrives before the postsynaptic spike (the "pre-post" regime). If the GABAergic input is blocked, presynaptic and postsynaptic spikes jointly cause a large membrane depolarization at the excitatory spine. After repetitive stimulation, the calcium concentration rises above the LTP threshold (Fig. $2 B$, top-right, red line), hence inducing LTP (Fig. 2B, bottom-right, red line). By contrast, if the GABAergic input arrives coincidentally with the presynaptic input, depolarization at the excitatory spine is attenuated by a negative current influx though the inhibitory synapse. As a result, the calcium concentration cannot reach the LTP threshold although it is still high enough to eventually cause LTD (Fig. $2 B$, right, black lines). Similarly, when the postsynaptic spike arrives at the spine before the presynaptic spike (the post-pre regime) in the absence of GABAergic input, the delayed presynaptic spike causes a slowing of the decay in the level of calcium concentration that may induce LTD (Fig. 2B, left, red lines). To the contrary, if the GABAergic input is provided simultaneously with the presynaptic input, the decay in the calcium concentration is sped up because of the hyperpolarization of the membrane potential at the excitatory spine by the inhibitory input. As a result, LTP is more likely to occur (Fig. 2B, left, black lines). Therefore, when a GABAergic input arrives in coincidence with a presynaptic excitatory input, the STDP time window changes its sign in both the pre-post and the post-pre stimulation regimes (Fig. 2A, lines).

A GABAergic effect on excitatory synaptic plasticity is also observed in CA1 pyramidal neurons (Hayama et al., 2013). In this case, the post-pre stimulation does not induce LTD unless GABA uncaging is conducted near the excitatory spine immediately before the postsynaptic spike arrives at the spine, whereas LTP is induced by the pre-post stimulation regardless of GABA uncaging (Fig. 2C, squares). Our model can also replicate these results. In the pre-post stimulation, the membrane potential of the spine shows strong depolarization due to positive feedback through the NMDA receptor, even if inhibitory current is delivered through GABA (Fig. 2D, top-right, red and black lines). Thus, LTP occurs after repetitive stimulation (Fig. 2D, bottom-right, red and black lines). By contrast, in the post-pre protocol, the effects of triggering LTP and LTD tend to cancel each other in the absence of GABAergic input, whereas LTD becomes dominant under the influence of GABAergic input (Fig. 2D, left, red and black lines, respectively).

In addition to the I-to-E effect, the E-to-E effect is also observed in the case of CA1 pyramidal neurons (Hayama et al., 2013). If GABA uncaging is performed immediately before postsynaptic firing, LTD is also observed in neighboring excitatory spines (Fig. 2E, right, point). This E-to-E heterosynaptic effect is not observed in the absence of GABAergic input (Fig. 2E, left, points). Correspondingly, in the model, excitatory current influx from a nearby synapse causes mild potentiation of calcium concentration in cooperation with inhibitory current influx, eventually inducing LTD (Fig. 2F, left, gray lines). Note that, in this E-to-E effect, interactions of signaling molecules or competition for resources at a later stage of synaptic plasticity may also play a dominant role (Hayama et al., 2013).

To check the parametric robustness of the model, we uniformly sampled the values of all the main parameters from fixed ranges, and studied the sensitivity of the model performance to each parameter, by calculating the performance distribution over the distributions of the other parameters (Fig. $3 A, B$ ). Even if the values of the parameters were perturbed by $20-100 \%$ from the 


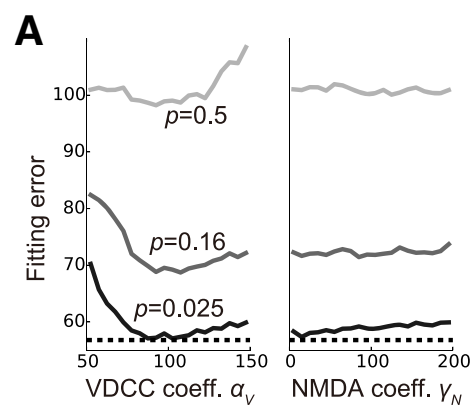

B

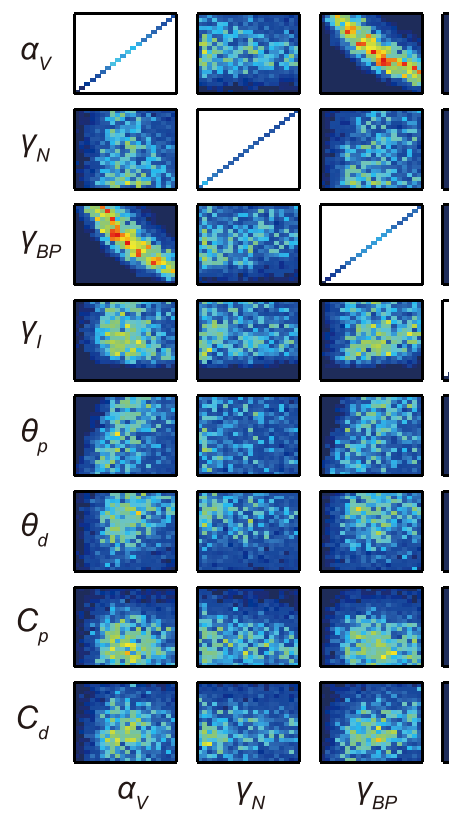

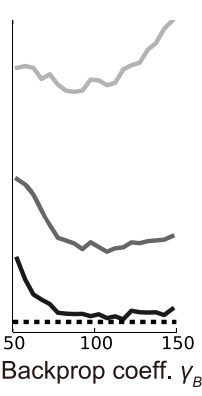
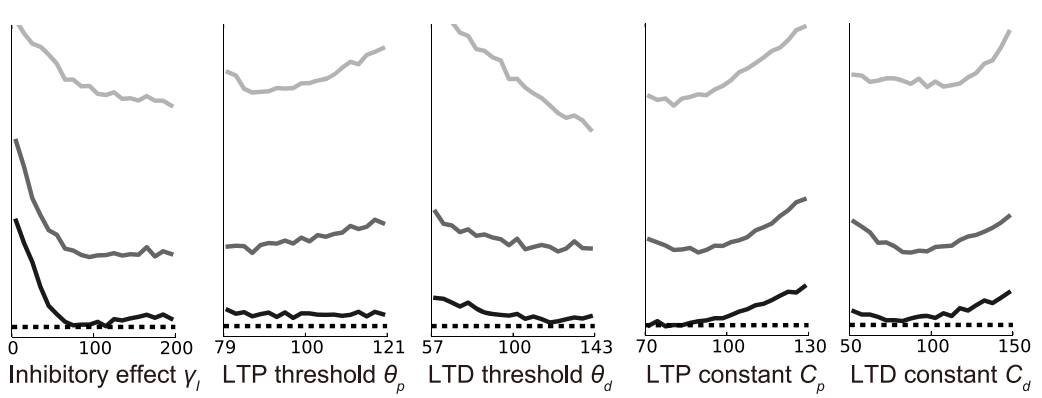

Fraction above the criteria
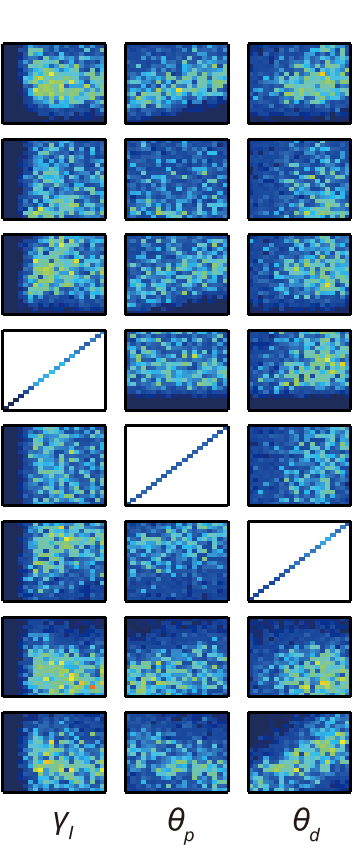
$\begin{array}{lllllll}0.0 & 0.03 & 0.06 & 0.09 & 0.12 & 0.15\end{array}$

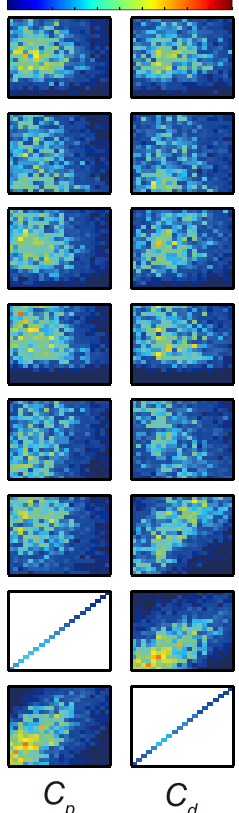

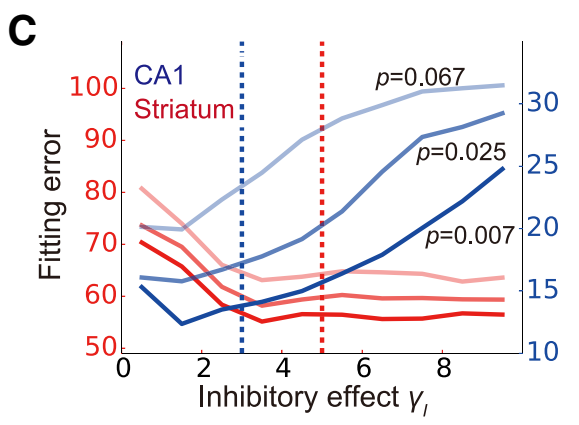

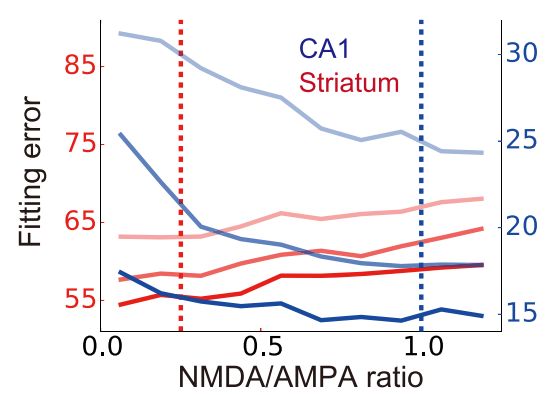

Figure 3. Parametric robustness of the h-STDP model. $A$, Fitting errors for the model of the striatum experiment at various values of model parameters. We conducted $10^{5}$ simulations by uniformly sampling all parameters within fixed ranges, and then calculated the median error (light gray line), 16 percentile error (dark gray line), and 2.5 percentile error (black line) for each parameter by marginalizing the other parameters. The $x$-axes show the range of perturbation used for all parameters, with the $100 \%$ values corresponding to the values used in Figure $2 A$ (Table 2 ). Dotted lines represent the fitting error for the parameter set used in Figure $2 A$. See Materials and Methods for the definitions of the parameters. The fitting error was calculated as $\sqrt{\sum_{d}^{N_{D}}\left(w_{\text {sim }}^{d}-w_{\text {data }}^{d}\right)^{2} / N_{D}}$, where $w_{\text {sim }}$ is the synaptic weight in the simulation, $w_{\text {data }}$ is the weight in the experiment (Paille et al., 2013), and $N_{D}$ is the number of data points $\left(N_{D}=64\right)$. $B$, Performance dependence on two-parameter relationships. For the same data depicted in $A$, we calculated the percentage of simulation trials that exceeded the fitting error criteria (error $<60.0$ ), under a given parameter pair. Dark blue points are parameter sets at which $0 \%$ of the simulations exceeded the criteria, whereas red points are the parameter sets where $15 \%$ did. The $x$ - and $y$-axes for each parameter are the same as in A.C, Comparisons of the parametric dependences of models fitted to the results from the striatum and CA1 experiments. The vertical dotted lines represent the values used in Figure 2. The NMDA/AMPA ratio in the bottom panel was calculated as $\gamma_{N} \tau_{N} / \gamma_{A} \tau_{A}$ at various values of $\gamma_{N}$. Here, the error for the striatum model was calculated over $10^{4}$ simulations, and the NMDA coefficient $\gamma_{\mathrm{N}}$ was uniformly sampled from $[0.0,0.25)$. Parameters for the CA1 model were sampled from the same ranges. Three lines with different shades represent the fitting error at $6.7,2.5$, and 0.7 percentiles.

original values (i.e., the values used in Fig. $2 A$ ), we obtained similar sizes of fitting errors to Figure $2 A$ in $\sim 2.5 \%$ of the simulations (Fig. 3A). Moreover, we found that anti-correlation between the VDCC coefficient and the backpropagation coefficient was crucial for replicating the experimental result (Fig. $3 B, \alpha_{\mathrm{V}}$ vs $\gamma_{\mathrm{BP}}$ and $\left.\gamma_{\mathrm{BP}} \mathrm{vs} \alpha_{\mathrm{V}}\right)$, as their product determined the effective amplitude of the calcium transient caused by a postsynaptic spike (Eq. $1+$ Eq. 3). A linear relationship between the LTP constant and LTD constant is also important for model fitting (Fig. $3 B, C_{p}$ vs $C_{d}$ and $C_{d}$ vs $C_{p}$ ). Notably, fitting the experimental data from the striatum requires a larger coefficient of the heterosynaptic inhibitory effect than the value required for fitting the data from CA1, and thus the striatum model depends on stronger inhibition than the CA1 model (Fig. 3C, top). This is consistent with the observation of strong inhibition in the striatum (Mallet et al., 2005). We also found that faithful reproducing of the CA1 experimental data crucially depended on a high NMDA/AMPA ratio, whereas the striatum model was rather robust against this ratio (Fig. $3 C$, bottom).

\section{Phase transitions underlying h-STDP}

In the previous section, we introduced a biophysical model to establish its relevance to the corresponding biological processes and obtain insight into the underlying mechanism. However, not all of the components of the model are necessary to reproduce the observed properties of h-STDP. We next provide a simple analytically tractable model to investigate the generality of the proposed mechanism.

To this end, we simplify the model to one in which the calcium level at a spine is directly modulated by the presynaptic, postsynaptic, and heterosynaptic activities, as given below: 
A

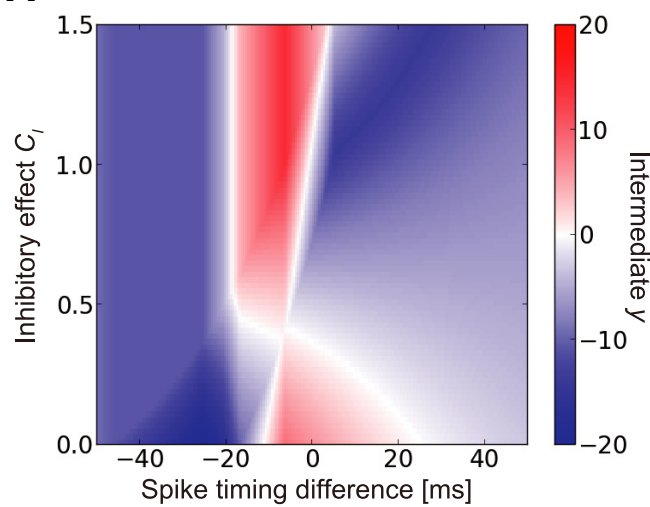

B

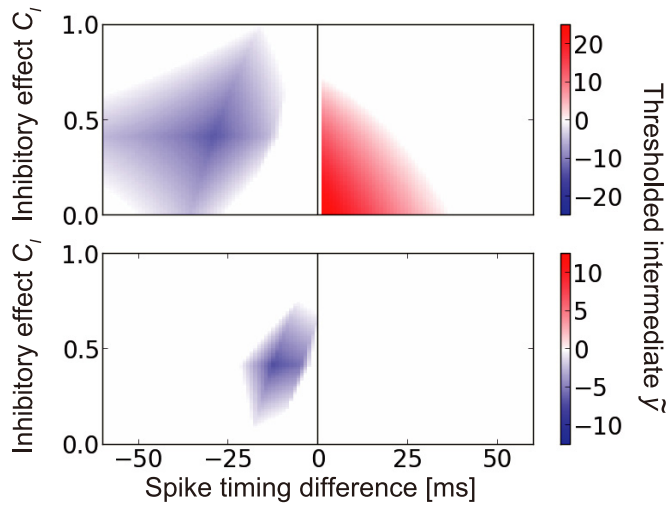

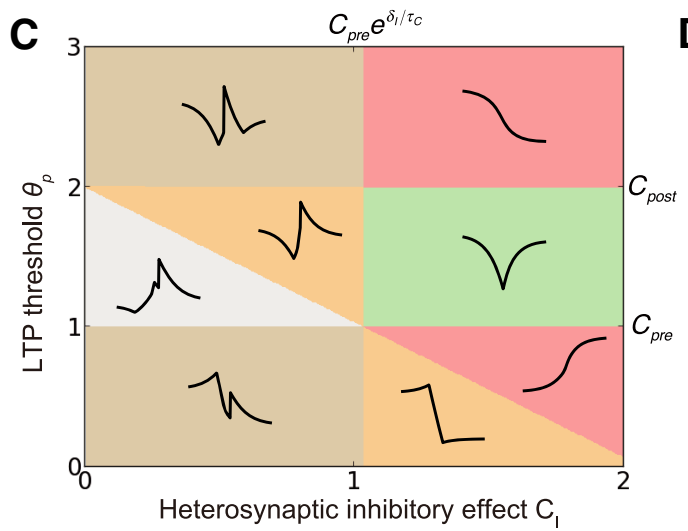

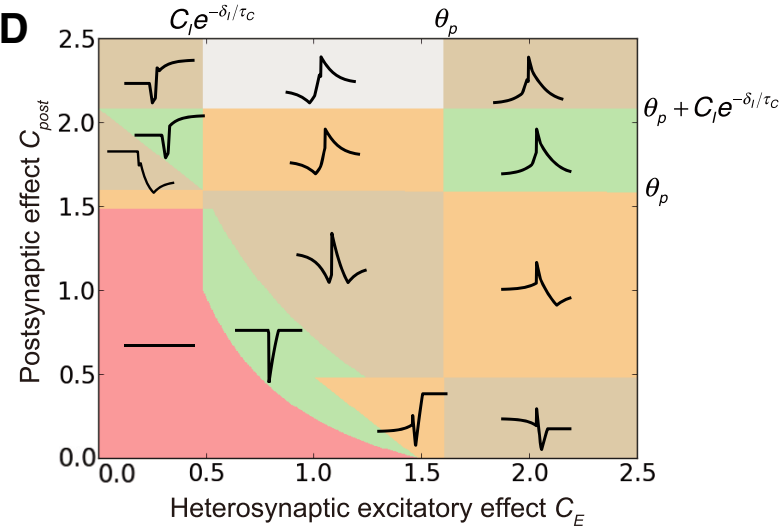

Figure 4. Phase transitions on the STDP time window in an analytical model of h-STDP. $A, B$, STDP windows at various values of heterosynaptic inhibitory effect $C_{1}$. $A$ corresponds to the striatum experiment and $\boldsymbol{B}$ corresponds to the CA1 experiment. $\boldsymbol{B}$, Top and bottom represent the stimulated and a neighboring spine, respectively. Note that values in $\boldsymbol{B}$ were calculated by $\tilde{y}=\operatorname{sgn}(y) \cdot[|y|-15]_{+}$to reflect the effect of thresholding. $C$, Phase diagram of the STDP time window calculated for the inhibitory effect $C_{1}$ and LTP threshold $\theta_{p}$. The colors show the number of local minima/maxima, whereas the lines are typical STDP time windows at each phase. The parameters written on the right side (top) of the panel represent the critical values of $\theta_{p}\left(C_{1}\right)$. $D$, The phase diagram calculated for the heterosynaptic excitatory effect parameter $C_{E}$ and the postsynaptic effect parameters $C_{\text {post }}$ at a fixed inhibitory effect $\left(C_{1}=0.5\right)$. See Materials and Methods, Reduced model.

$$
\begin{gathered}
\frac{d C_{i}(t)}{d t}=-\frac{C_{i}(t)}{\tau_{C}}+C_{p r e} X_{i}(t)+C_{p o s t}\left[1+g_{C}\left(C_{i}(t-\Delta t)\right)\right] \\
\times X_{p o s t}(t)-C_{I} \sum_{j \in \Omega_{i}^{I}} X_{j}^{I}\left(t-d_{I}\right)+C_{E} \sum_{j \in \Omega_{i}^{E}} X_{j}^{E}\left(t-d_{E}\right) .
\end{gathered}
$$

Here, $C_{i}(t)$ represents the $\mathrm{Ca}^{2+}$ concentration at spine $i, X_{i}$, and $X_{\text {post }}$ represent presynaptic and postsynaptic spikes respectively, $d_{\mathrm{I}}$ and $d_{\mathrm{E}}$ are heterosynaptic delays, and $\Omega_{i}^{\mathrm{I}}$ and $\Omega_{i}^{\mathrm{E}}$ are the sets of neighboring inhibitory and excitatory synapses (for details of the model, see Materials and Methods, Reduced model). Despite its simplicity, the model can qualitatively reproduce the heterosynaptic effects observed in striatal and CA1 neurons, although the quantitative accuracy is degraded (Fig. $4 A$ and $B$, respectively). Importantly, the reduced model provides further analytical insights into the phenomena.

Let us consider how the inhibitory effect parameter $C_{\mathrm{I}}$ controls the I-to-E heterosynaptic effect observed in the CA1 experiment. If we characterize the shape of the STDP time windows by the total number of their local minima/maxima, the parameter space can be divided into several different phases (Fig. 4C). If the LTP threshold $\theta_{p}$ satisfies $C_{\text {pre }}<\theta_{p}<C_{\text {post }}$, a Hebbian type STDP time window appears when the strength of heterosynaptic inhibitory effect $C_{\mathrm{I}}$ satisfies $\left(C_{\text {post }}-\theta_{p}\right) e^{\delta_{I} / \tau_{C}}<C_{I}<C_{\text {pre }} e^{\delta_{I} / \tau_{C}}$ (Fig. $4 C$, top, orange region; see Materials and Methods for details of the analysis). Here, we defined $\delta_{\mathrm{I}}$ as the spike-timing difference between the inhibitory spike and the presynaptic or postsynaptic spikes in the pre-post or the post-pre stimulation protocols, respectively. If $C_{\mathrm{I}}$ is larger than $C_{\mathrm{pre}} \exp \left(\delta_{\mathrm{I}} / \tau_{C}\right)$, a strong inhibitory effect causes LTD, even in the pre-post regime (Fig. $4 C$, green region), whereas LTD in the post-pre regime is suppressed when $C_{\mathrm{I}}$ is smaller than
$\left(C_{\text {pre }}-\theta_{p}\right) \exp \left(\delta_{I} / \tau_{C}\right)$ (Fig. $4 C$, gray region). Thus, the heterosynaptic LTD observed in Figure $2 C$ can be represented as the phase shift from the gray-colored region to the orange-colored region in Figure $4 C$, which is due to the change in the inhibitory effect $C_{\mathrm{I}}$. This analysis further confirms the condition for inducing heterosynaptic LTD where the heterosynaptic spike-timing difference $\delta_{\mathrm{I}}$ should be smaller than the timescale of $\mathrm{Ca}^{2+}$ dynamics $\tau_{C}$ (Hayama et al., 2013). This is because $\delta_{l}<\tau_{C} \log \left(\frac{C_{l}}{C_{\text {post }}-\theta_{p}}\right)$ is necessary for a significant heterosynaptic LTD, and $C_{\mathrm{I}}$ is typically smaller than $C_{\text {post }}$ and $\theta_{p}$. In addition, heterosynaptic suppression of the pre-post LTP (green region) is very unlikely to occur, as it is necessary for $C_{\mathrm{I}}$ to be larger than $C_{\mathrm{pre}} \exp \left(\delta_{\mathrm{I}} / \tau_{C}\right)$. This condition is difficult to satisfy even if $\delta_{\mathrm{I}}=0$, because the heterosynaptic effect on $\mathrm{Ca}^{2+}$ dynamics in the spine is expected to be smaller than the homosynaptic effect (i.e., $C_{I}<C_{\text {pre }}$ ). A similar analysis is possible for E-to-E interactions, although the phase diagram becomes complicated in this case (Fig. 4D; see Materials and Methods).

These analyses reveal that the heterosynaptic effects are always observable when the parameters of the calcium dynamics fall within a certain region of the parameter space, and underscore the robustness of h-STDP in our framework.

\section{h-STDP induces the detailed dendritic E/I balance at dendritic hotspots}

The results described so far suggest that the proposed model gives a good approximation of h-STDP. To investigate the possible functions of h-STDP, we next examined how this h-STDP rule shapes the synaptic organization on the dendrite of a simulated 
A

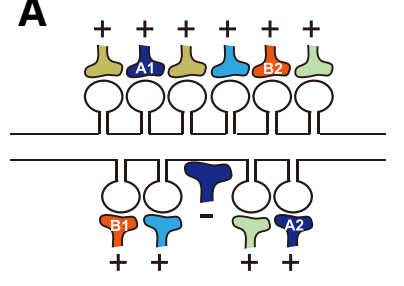

B

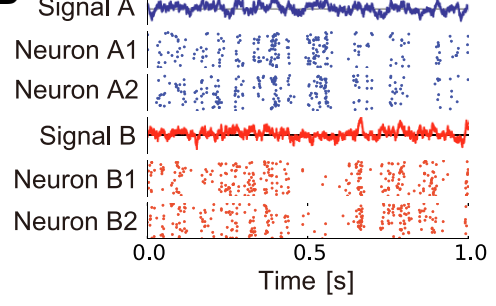

D
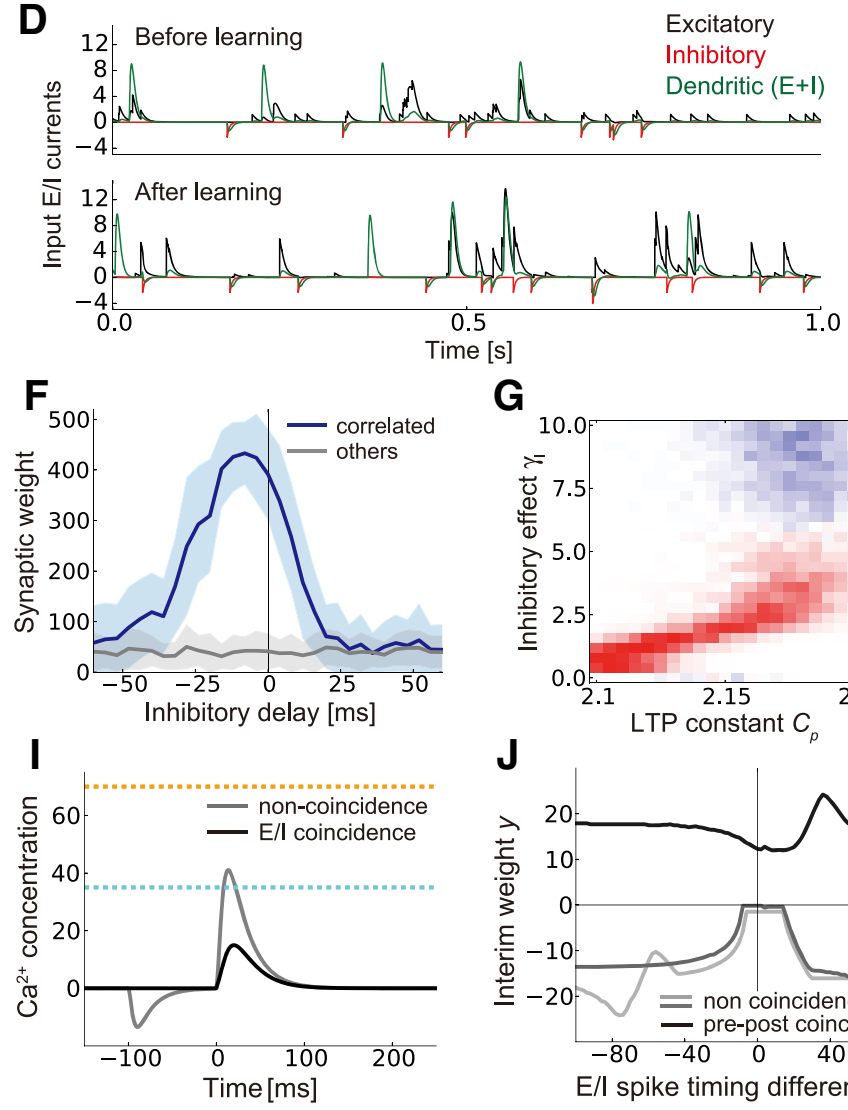

G
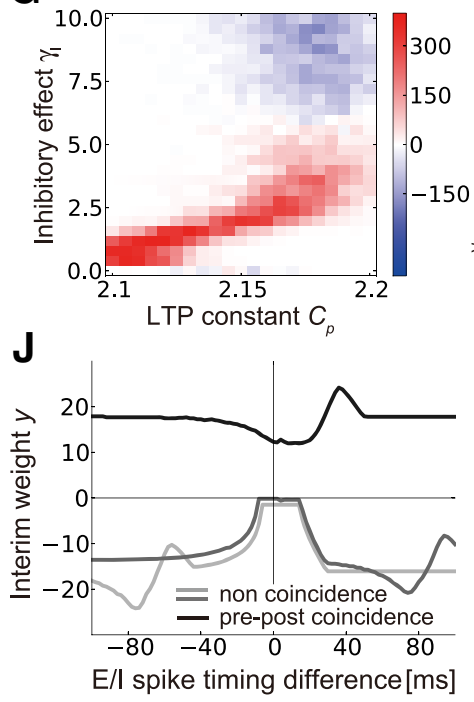

C

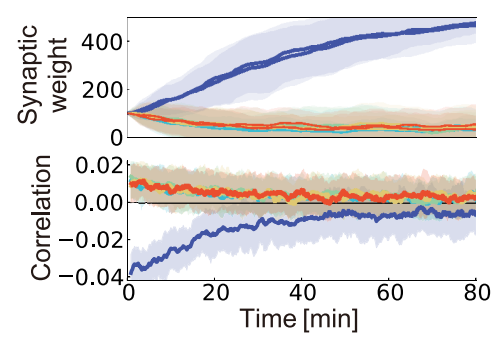

E

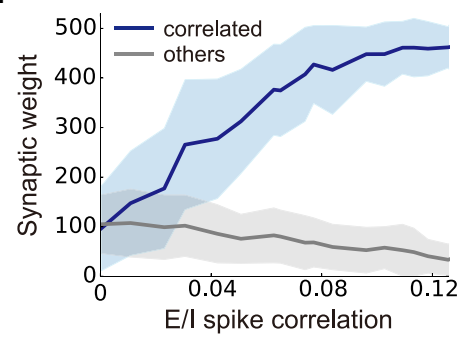

H

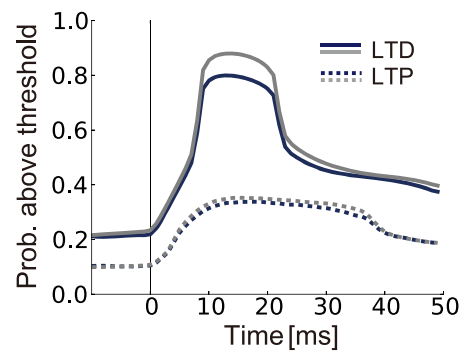

Figure 5. Emergence of detailed dendritic excitatory/inhibitory balance by h-STDP. A, A schematic figure of a dendritic hotspot model. The shaft synapse represents an inhibitory input. Colors represent the spike correlations between synaptic inputs. $\boldsymbol{B}$, Examples of correlated spike inputs. Each raster plot was calculated from 50 simulation trials. $C$, Changes in the synaptic weight, $w$ (top), and the correlation between the dendritic membrane potential and hidden signals (bottom), under h-STDP. The blue lines represent the dynamics of synapses correlated with the inhibitory input. D, Traces of excitatory and inhibitory inputs before and after learning. Positive correlations between excitatory and inhibitory currents before learning (top, black and red lines) shift to large negative correlations after learning (bottom, lines). The excitatory and inhibitory input currents were approximately defined as $u_{E}(t)=\frac{1}{w_{o}^{E}} \sum_{i=1}^{10} w_{i}\left[\gamma_{A} x_{i}^{A}(t)+\gamma_{N} g_{N}\left(u_{i}\right) x_{i}^{N}(t)\right]$ and $u_{I}(t)=-\gamma_{G} x_{G}(t)$, respectively. In the graph, we scaled the inhibitory current twofold for illustration purposes. The green lines represent the dendritic membrane potential $u_{b}(t)$. The learning was performed using the same parameter values as in the simulation depicted in $\boldsymbol{C}$. $\boldsymbol{E}$, Synaptic weight dependence on excitatory/inhibitory spike correlation. We changed the correlation by modulating the sensitivity of inhibitory activity to the hidden signal, while keeping the inhibitory firing rate fixed at $r_{1}=9.2 \mathrm{~Hz}$. The spike correlation was calculated by taking 25 millisecond bins. $\boldsymbol{F}$, Synaptic weight change at the excitatory synapses correlated with the inhibitory inputs (blue), and at other synapses (gray), at various inhibitory delays. Error bars in $\boldsymbol{C}, \boldsymbol{E}$, and $\boldsymbol{F}$ represent $S D$ s over 50 simulation trials. $\mathbf{G}$, The relative weight changes $w_{\mathrm{R}}$ calculated for various parameters. We defined $w_{\mathrm{R}}$ as $\left\langle w_{i}^{E}\right\rangle_{i \in \text { corr }}-\left\langle w_{i}^{E}\right\rangle_{i \in \text { un-corr }}$, where "corr" represents a set of excitatory synapses correlated with the inhibitory synapse, and "un-corr" stands for uncorrelated ones. The weights were calculated by taking an average over 10 simulations. $\boldsymbol{H}$, The probability of a LTP/LTD occurrence after a presynaptic spike calculated from a simulation. The lines represent the mean LTP/LTD probabilities at excitatory synapses correlated with the inhibitory input (blue lines) and other synapses (gray lines), respectively. $I, J$, Results from single-spike simulations. The E/I coincidence prevents the LTD effect due to the pre-spike $(I)$, without affecting the LTP effect due to pre-post coincidence $(J)$. In $I$, the inhibitory spikes were provided at $t=0$ for the black line and $t=-100 \mathrm{~ms}$ for the gray line, with the excitatory presynaptic spike being given at $t=0$ in both lines. Similarly, in $J$, the postsynaptic spikes were provided at $t=-75$ (light-gray), 0 (black), and $+75 \mathrm{~ms}$ (dark-gray), while the presynaptic spike was given at $t=0$ in all lines.

neuron. We first considered a model of a dendritic hotspot (Jia et al., 2010) that receives 10 excitatory inputs and one inhibitory input (Fig. 5A), because the heterosynaptic effect is typically confined within $10 \mu \mathrm{m}$ of the synapse (Hayama et al., 2013). Excitatory inputs are organized into five pairs, with each pair of excitatory synapses receiving correlated inputs (Fig. 5B; see Materials and Methods, Dendritic hotspot model). In addition, the inhibitory input is correlated with one excitatory pair (Fig. $5 \mathrm{~A}$, A1 and A2). Here, we assumed that postsynaptic activity follows a Poisson process with a fixed rate, because the influence of a single hotspot on the soma is usually negligible. In addition, we neglected the effect of morphology and supposed that the heterosynaptic interaction occurs instantaneously within the hotspot. In this configuration, surprisingly, excitatory synapses correlated with the inhibitory input are potentiated, while other excitatory synapses experience minor depression (Fig. 5C, top). 
As a result, the dendritic membrane potential of the branch becomes less correlated with all the hidden signals, because the strong negative correlation with the blue signal is cancelled by the potentiated excitatory inputs, whereas weak positive correlations with other signals are diminished due to LTD at the corresponding excitatory synapses (Fig. $5 C$, bottom). Although input spikes to the hotspot is sparse and stochastic, the traces of excitatory and inhibitory currents shift toward the detailed balance after learning (Fig. 5D). A spike correlation between excitatory and inhibitory inputs is crucial for this potentiation of excitatory synapses, but in the model a small correlation is sufficient to produce a significant change in the synaptic weight (Fig. 5E). Moreover, this GABA-driven potentiation is only observable when inhibitory activity is precisely correlated with excitatory activities, and becomes larger when the inhibitory spike precedes excitatory spikes, rather than when it follow them (Fig. $5 F$ ). We also found that, when the heterosynaptic inhibitory effect $\gamma_{\mathrm{I}}$ is large enough to cause a strong hyperpolarization at nearby synapses, depression is observed at correlated excitatory synapses (Fig. $5 G$, blue area) instead of potentiation (Fig. $5 G$, red area). However, as can be seen in Figures 3 and 4, such a large inhibitory effect does not reproduce the STDP experiments, especially the data from the CA1 pyramidal neurons, and is thus unlikely to be observed in the actual brain. These results indicate that h-STDP induces a dendrite-specific temporally precise E/I balance by potentiating excitatory synapses that are correlated with inhibitory synapses.

To reveal the underlying mechanism of this E/I balance generation, we used the simulation data to calculate the probability of the calcium level reaching above the LTD/LTP thresholds after a presynaptic spike. The probabilities of LTP occurrences show similar trajectories after a presynaptic spike, regardless of whether the presynaptic activity is correlated with inhibitory input or not (Fig. $5 \mathrm{H}$, blue and gray dotted lines, respectively). However, the peak probability of LTD occurrence is significantly lower for spines that are correlated with inhibitory inputs (Fig. $5 H$, blue vs gray solid lines), although in both cases the probability goes up after the presynaptic spike. This asymmetry between LTP and LTD is consistent with the following interpretation: LTD is mainly caused when the presynaptic neuron fires at a low firing rate and the postsynaptic neuron remains silent, both in the experiments (Malenka and Bear, 2004) and in our model (Fig. 5I, gray line). However, if an inhibitory input arrives at a nearby dendrite in coincidence with excitatory activity, the calcium boost caused by the excitatory presynaptic input is attenuated by the heterosynaptic inhibitory effect (Fig. 5I, black line). As a result, LTD is shunted by correlated inhibitory inputs. On the other hand, LTP is mainly caused by coincident presynaptic and postsynaptic spikes, which induce a large increase in calcium that overwhelms the heterosynaptic inhibitory effect. Thus, LTP at correlated excitatory synapses is not compromised by inhibitory activity at a nearby site (Fig. $5 J$ ). Therefore, correlated spines tend to be potentiated overall.

To evaluate the generality of the observed dendritic E/I balance, we extended the model to a two-layered single cell (Poirazi et al., 2003) by modeling each branch with one dendritic hotspot (Fig. 6A; see Materials and Methods, Two-layered neuron model), and investigated the dendritic organization of synaptic weight changes by h-STDP. In the simulation, we introduced a $10 \mathrm{~ms}$ delay between the excitatory and inhibitory stimulation (Froemke, 2015). Even in this case, when the dendritic branches of a postsynaptic neuron receive inputs from various neurons with different selectivity, each dendritic hotspot shapes its excitatory synaptic organization according to the selectivity of its inhibitory input (Fig. $6 B, D$; the frame colors in $B$ represent the inhibitory selectivities). As a result, the excitatory synapses on the dendritic tree become clustered, as observed in previous experiments (Kleindienst et al., 2011; Takahashi et al., 2012). Note that, in our model, this clustering of excitatory synapses is caused by common inhibitory inputs, instead of direct interactions between excitatory spines.

To further clarify the importance of heterosynaptic interaction, we next compared the results of h-STDP with that of learning under the standard STDP (Song et al., 2000). In the model, we introduced a branch-specific homeostatic plasticity to induce competition among nearby synapses (see Materials and Methods for the details of the standard STDP model). In the standard STDP model, all dendritic branches developed similar synaptic distributions regardless of the differences in local inhibitory selectivity (Fig. 6C; the beige signal is learned in this example), because one of the hidden signals is captured by chance through self-organization (Song et al., 2000). As a result, under the standard STDP rule the synaptic organizations of branches remain akin to each other (Fig. $6 E$, gray line). By contrast, under the h-STDP rule, each dendritic branch acquires a synaptic structure according to its local inhibitory input, and individual dendritic branches become dissimilar from one another (Fig. 6E, black line).

We further investigated the possible function of this synaptic organization in information processing. To this end, we consecutively presented the five stimuli to the two-layered neuron model (Fig. 6F). Before learning, the neuron showed an almost constant response to the stimulation, with a small dip at the change points between each of the five stimulations (Fig. 6F, top). By contrast, after learning, the neuron showed transient bursting activity immediately after the onset of the new $500 \mathrm{~ms}$ stimulus window, and then rapidly returned to an almost silent state (Fig. $6 F$, middle). Hence, by h-STDP, a neuron can acquire sensitivity toward abrupt changes in stimuli (Fig. $6 F$, bottom, $G$ ). This sensitivity vanished if the selectivities of inhibitory synapses were randomly shuffled, suggesting the importance of the balance at each dendrite (Fig. $6 H$ ). This result indicates that although the detailed E/I balance at each dendrite has a small overall effect to the somatic membrane dynamics; the collective effect from all dendritic branches has a significant impact on the postsynaptic activity.

\section{The effect of dendritic spikes}

Previous studies on dendritic computation reveal the potential importance of dendritic spikes in synaptic plasticity (Smith et al., 2013; Kastellakis et al., 2016). Although we mainly consider a regime within a low firing rate $(\sim 5 \mathrm{~Hz})$, dendritic spikes may occur due to strong input spike correlation. We therefore extended the model discussed in the previous section by including dendritic spikes.

Here, we focus on $\mathrm{Na}^{+}$spikes that are typically localized within a dendritic branch, not global $\mathrm{Ca}^{2+}$ spikes (London and Häusser, 2005), as we are interested in branch-specific synaptic organization. We modeled dendritic spikes as an excitatory heterosynaptic interaction with thresholding, and then added the interaction to the dendritic hotspot model (see Materials and Methods, Dendritic hotspot model). When the amplitude of a dendritic spike is comparable to the amplitude of backpropagating spike, the synapses set their weights differently, depending on the threshold of a dendritic spike. When the spike threshold is very low, the relative weight difference between the correlated and uncorrelated excitatory synapses converges to zero, because 


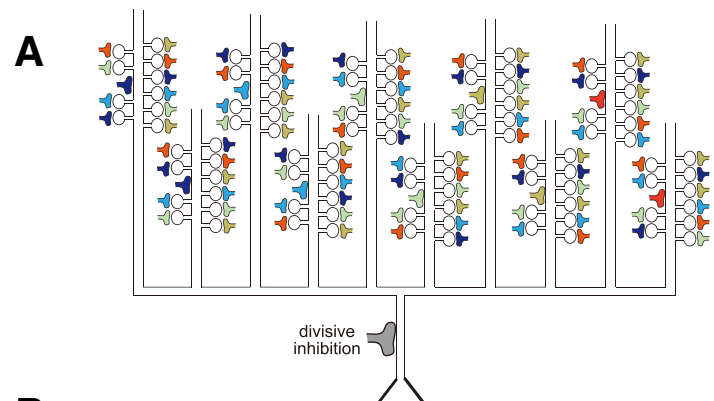

B
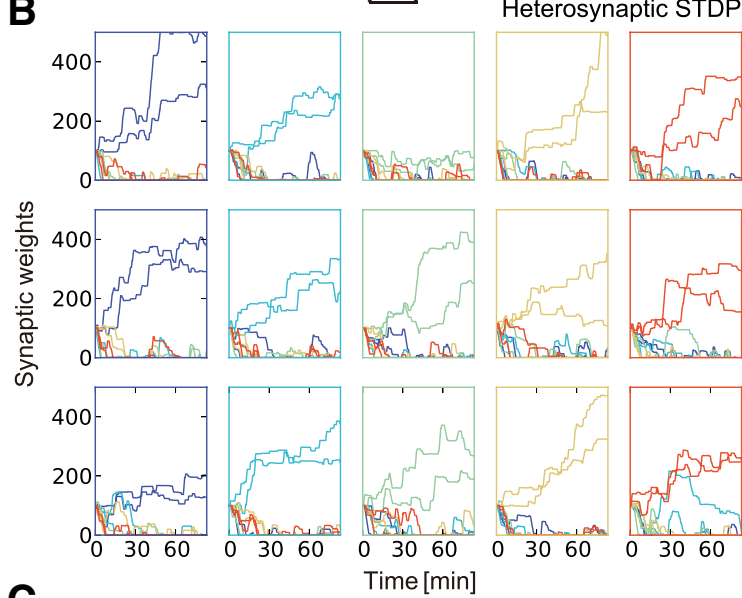

C
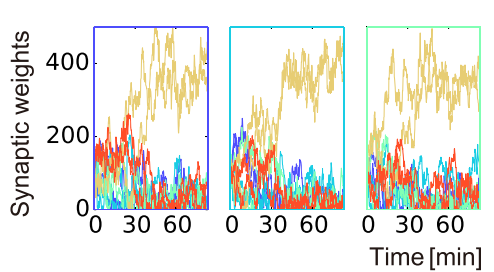

D
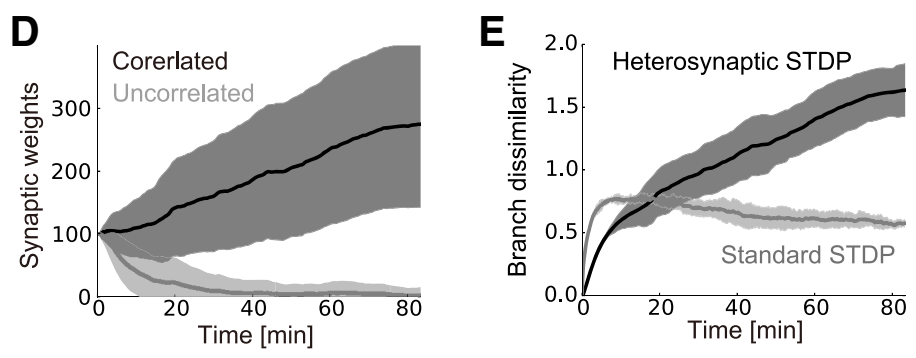

$\mathbf{F}$
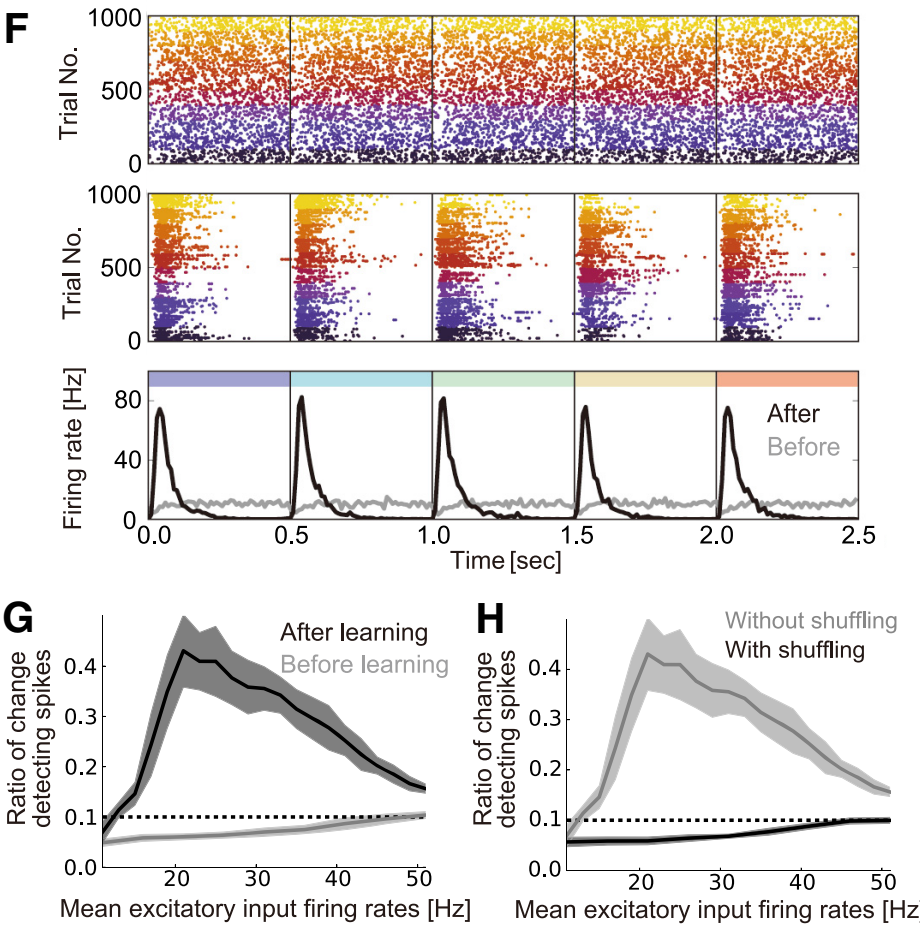

Figure 6. Detailed dendritic excitatory/inhibitory balance in a two-layered single cell model. $A$, A schematic illustration of the single cell model. The actual model has 100 dendritic branches each receiving 10 excitatory inputs and 1 inhibitory input. As in Figure $5 A$, the inhibitory inputs are represented by shaft synapses. $\boldsymbol{B}$, Examples of synaptic weight change at each branch. The color of the frames represents the selectivity of the inhibitory input to the branch. Each row represents a different simulation trial. $C$, An example of synaptic weight change at each branch under the standard STDP rule. As in $\boldsymbol{B}$, the frame colors represent the local inhibitory selectivity. $\boldsymbol{D}$, The mean synaptic weight dynamics of synapses correlated with the local inhibitory inputs and other synapses under h-STDP. $\boldsymbol{E}$, Development of the branch dissimilarity under the two learning rules. Dissimilarity was given as $\frac{1}{K(K-1) w_{o}} \sum_{k}^{K} \sum_{k^{\prime} \neq k}^{K} \sqrt{\frac{1}{N_{b}} \sum_{i}^{N_{b}}\left(w_{k, i}-w_{k^{\prime}, i}\right)^{2}}$. $\boldsymbol{F}$, Raster plots of output spikes before (top) and after (middle) learning, and their firing rate dynamics (bottom), taken from 100 trials each for 10 simulated neurons. The colors of the spikes in the raster plots represent results from different simulation trials. The black vertical lines represent the points of change in excitatory inputs, and the horizontal colored bars at the top of the bottom panel correspond to the colors of the presynaptic neurons active in each period. $G$, The ratio of change detecting spikes before and after learning. The ratio was defined as the fraction of spikes occurring within $50 \mathrm{~ms}$ of a change in stimuli to the total number of spikes. On the $x$-axis, in addition to the mean excitatory input firing rates, the mean inhibitory input firing rates were also modulated from 50 to $210 \mathrm{~Hz}$ correspondingly, to maintain the $\mathrm{E} / \mathrm{I}$ balance of the input. $\boldsymbol{H}$, The ratio of change detecting spikes with/without inhibitory shuffling. The gray line is the same with the black line in $\boldsymbol{G}$. In the black line, selectivities of inhibitory synapses were randomly shuffled to break the $E / I$ balance at each dendrite. We introduced a $10 \mathrm{~ms}$ delay between the excitatory and inhibitory stimulus, both during learning $(\boldsymbol{B}-\boldsymbol{E})$ and in the change detecting task $(\boldsymbol{F}-\boldsymbol{H})$. The averages in $\boldsymbol{D}, \boldsymbol{E}, \boldsymbol{G}$, and $\boldsymbol{H}$ were taken over 10 simulation trials.

all excitatory synapses are potentiated in this regime. In contrast, under conditions of a very high threshold, dendritic spikes rarely occur, so the correlated synapses are only moderately potentiated, as in the control (Fig. 7A; the dotted line represents the control). Notably, when four to five spikes from synapses with the unit weight $\left(w=w_{\mathrm{o}}\right)$ are sufficient to generate dendritic spikes, the relative weight differences becomes larger than the control, as the dendritic spikes selectively strengthen such synapses that are moderately potentiated by h-STDP (Fig. 7A). This effect is especially significant when inhibitory spikes are delayed by $5-10 \mathrm{~ms}$ on average (Fig. $7 B$ ). These results suggest that local dendritic spikes stabilize the synaptic weight structure generated through heterosynaptic STDP.

\section{h-STDP explains the critical period plasticity of binocular matching}

The results so far indicate that h-STDP induces GABA-driven reorganization of synaptic weights, which in turn enriches dendritic computation such as in the enhanced sensitivity to detect changes in input activity. To investigate its relationship with developmental plasticity, we next consider a model of critical period plasticity in binocular matching (B. S. Wang et al., 2010, 2013). In mice, 1 week after the eye opening, binocular neurons in V1 typically exhibit different orientation selectivity for inputs from the two eyes. Nevertheless, after another 2 weeks, the selective orientations for each eye become closer, and eventually they almost coincide with each other (B. S. Wang et al., 2010). More- 
A

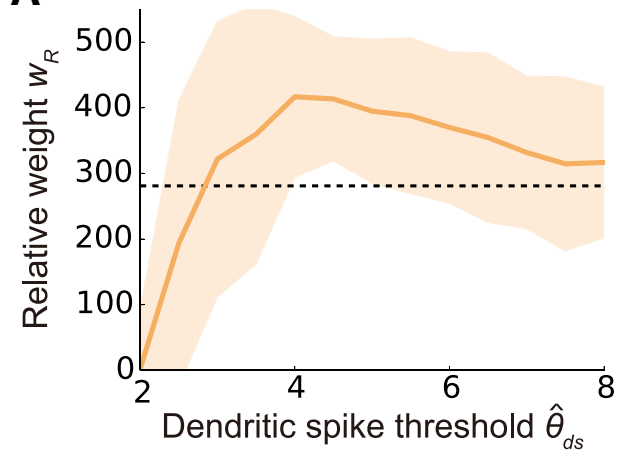

B

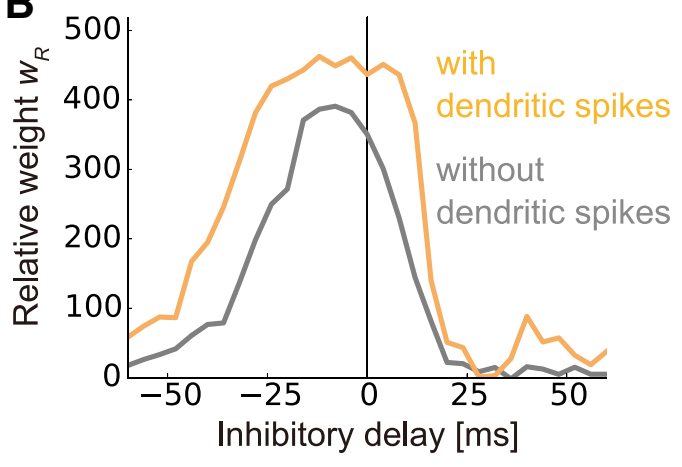

Figure 7. Dendritic spike stabilizes the detailed dendritic balance. $\boldsymbol{A}$, Relative synaptic weight $w_{\mathrm{R}}$ obtained at various dendritic spike thresholds for an inhibitory delay of 10 ms. The relative synaptic weight was defined as the difference between the weights of the synapses correlated with the inhibitory input and the other excitatory synapses, as in Figure $5 G$. The $x$-axis is normalized as $\hat{\theta}_{d s}=\theta_{d s} / w_{o}$, and the dotted line shows the value without dendritic spikes (control). $\boldsymbol{B}$, Relative synaptic weight $w_{\mathrm{R}}$ for various inhibitory delays at $\hat{\theta}_{d s}=4.0$. The gray line is the same as the difference between the two lines in Figure $5 F$.

\section{A}

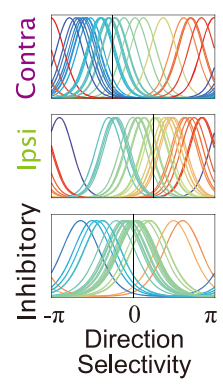

D
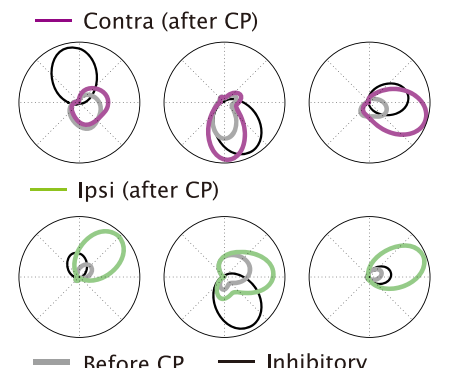
Contra Inhibitory Ipsi inputs inputs inputs

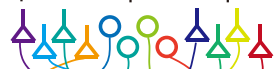
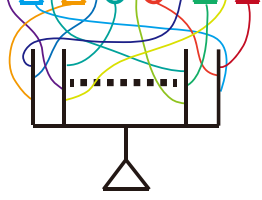$$
\text { (1) }
$$

B

\section{E}

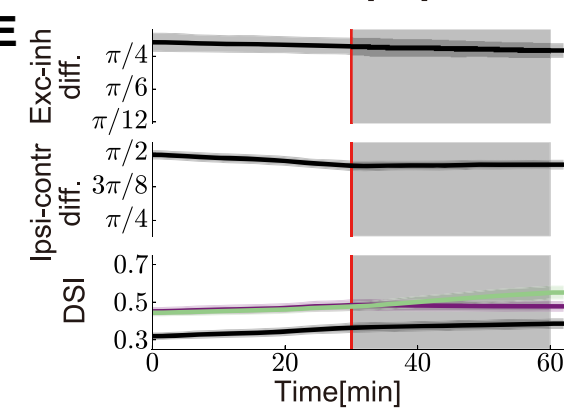

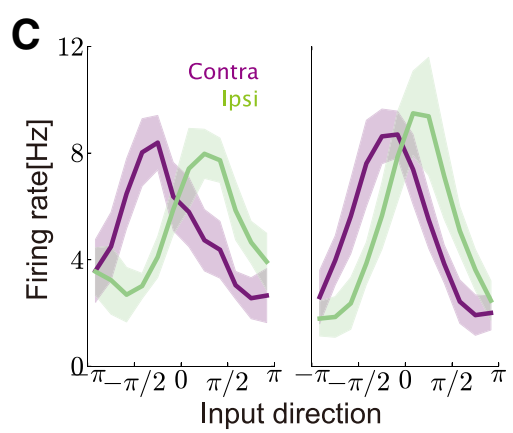

$\mathbf{F}$

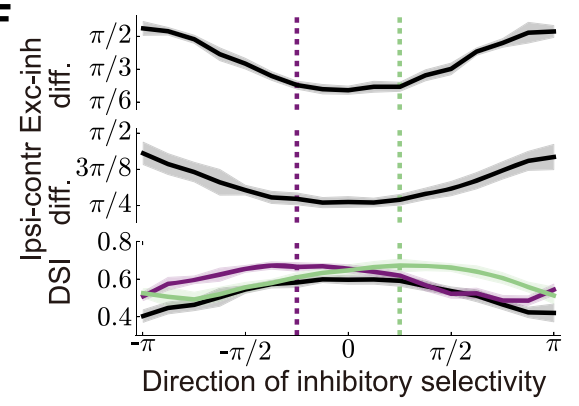

Figure 8. h-STDP can trigger binocular matching. $A$, Left, Direction selectivity of input neurons. In the model, as depicted by the black vertical lines, the majority of excitatory input neurons from the contralateral (ipsilateral) eye are selective for directions around $\theta=-\pi / 4(\pi / 4$, whereas inputs from the inhibitory neurons are weakly selective for $\theta=0$. Right, A schematic figure of the model configuration. Each dendritic branch receives inputs from both ipsilateral and contralateral-driven excitatory neurons, and also from inhibitory neurons. $\boldsymbol{B}$, Top, Difference between the mean excitatory direction selectivity and inhibitory direction selectivity in each branch. Middle, Difference between the mean ipsilateral-driven excitatory direction selectivity and the mean contralateral-driven excitatory direction selectivity over all synapses on the neuron. Bottom, DSI calculated for contralateral inputs (purple), ipsilateral inputs (light green; hidden under the purple line), and binocular inputs (black). See Materials and Methods, The model of binocular matching for details of the evaluation methods. Red vertical lines represent the timings for the introduction of inhibitory inputs. Throughout Figure 8, the error bars are SD over 10 simulation trials. C, Firing responses of the neuron for monocular inputs, immediately after the initiation of inhibitory inputs (left; $t=30 \mathrm{~min}$ ) and after the learning (right; $t=60 \mathrm{~min}$ ). $D$, Examples of the direction selectivity of three representative branches before ( $\mathrm{gray}$ lines; $t=0 \mathrm{~min}$ ) and after (purple/light $\mathrm{green}$ lines; $t=60 \mathrm{~min}$ ) the learning. Black lines represent the selectivity of the inhibitory input to the branch. $\boldsymbol{E}$, Behavior in the monocular deprivation model. In the shadowed areas, contralateral-driven inputs were replaced with rate-fixed Poisson inputs to mimic monocular deprivation. The ordinates are the same with $\boldsymbol{B}$. $\boldsymbol{F}$, Synaptic weights development at different mean inhibitory selectivity. Ordinates are the same as in $\boldsymbol{B}$, and the values were calculated at $t=60 \mathrm{~min}$. The purple and green vertical dotted lines are the mean selectivity of contralateral and ipsilateral excitatory inputs, respectively.

over, this binocular matching is disrupted by accelerating inhibitory maturation (B. S. Wang et al., 2013). Thus, the activity of inhibitory neurons plays a decisive role in shaping binocular matching, in addition to Hebbian plasticity at excitatory synapses.

We modeled this process with the two-layered single cell model introduced in Figure 6 (Fig. 8A, right; see Materials and Methods, The model of binocular matching). The input spike trains were modeled as rate-modulated Poisson processes driven by a circular variable $\theta$, which corresponds to the direction of moving visual stimuli. We assumed the following: (1) inputs from ipsilateral and contralateral eyes already have some weak orientation selectivity at the eye-opening (B. S. Wang et al., 2010; Espinosa and Stryker, 2012), (2) inhibitory cells are driven by both ipsilateral and contralateral eyes (Yazaki-Sugiyama et al., 2009; Kuhlman et al., 2011), and (3) the average orientation selectivity of inhibitory inputs fall between the orientation selectivity for ipsilateral and contralateral excitatory inputs (Fig. $8 \mathrm{~A}$, left). This last assumption has not yet been supported by experimental evidence, but if inhibition is provided by neighboring 
interneurons, these inhibitory neurons are likely to be driven by similar sets of feedforward excitatory inputs to those driving the output neuron. For mathematical convenience, we consider direction selectivity instead of orientation selectivity, but the same argument holds for the latter.

In the simulation, we first ran the process without inhibition, and then introduced GABAergic inputs after a while (Fig. $8 B, E$, red vertical lines represent the starting points of inhibitory inputs), because maturation of the inhibitory neurons typically occurs in a later stage of the development (Hensch, 2005). Upon the introduction of inhibition, the mean preferred direction selectivity of excitatory synapses in each branch converges to that of the local inhibition, because of heterosynaptic plasticity (Fig. 8B, top; see Materials and Methods for details of evaluation methods), although the synaptic weight development was biased toward the overall direction selectivity of the postsynaptic neuron (Fig. $8 D$; the bias is toward the zero-degree direction). This dendritic E/I balancing reduces the difference between the direction selectivity of ipsilateral and contralateral inputs on average, because both become closer to the selectivity of the inhibitory input (Fig. $8 B$, middle). As a result, the binocular direction selectivity is strengthened (Fig. 8B, bottom), and the responses for monocular inputs approximately coincide with each other (Fig. $8 C$, right). Deprivation of the contralateral inputs immediately after the introduction of inhibition blocks binocular matching (Fig. $8 E$ ), in accordance with the experimental data (B. S. Wang et al., 2010).

Precocious GABA maturation has been reported to disrupt binocular matching (B. S. Wang et al., 2013). Our model suggests that the disruption is possibly related to the violation of the third assumption in the model. When the mean inhibitory direction selectivity is substantially different from the ipsilateral and the contralateral direction selectivity (Fig. $8 F$, at the parameter regions outside of the area surrounded by purple and green lines), h-STDP does not work effectively (Fig. $8 F$, top), and the difference between ipsilateral and contralateral inputs is not reduced (Fig. 8F, middle). As a result, the binocular direction selectivity is not improved by learning (Fig. $8 F$, bottom). These results indicate that the rate of maturation of GABA inputs and their effect on h-STDP are an important part of the underlying mechanisms of binocular matching in critical period plasticity.

\section{Discussion}

In this study, we first showed that a calcium-based plasticity model robustly captures several characteristics of the plasticityrelated interactions between neighboring synapses that occur on a millisecond timescale; this was accomplished by the introduction of heterosynaptic interaction terms (Figs. 2-4). On the basis of this proposed model, we next investigated the possible functions of h-STDP. This study revealed that h-STDP causes the detailed dendritic E/I balance on dendritic hotspots (Figs. 5-7), which is beneficial for detecting changes in input activity (Fig. 6). Furthermore, we found that h-STDP can induce binocular matching upon GABA maturation, and can support an accurate input estimation (Fig. 8).

\section{Experimental predictions}

This study provides three experimentally testable predictions. First, our results provide a hypothesis for synaptic organization on the dendritic tree. Excitatory synaptic inputs to a dendritic hotspot often show correlated activities (Kleindienst et al., 2011; Takahashi et al., 2012). Our results indicate that an inhibitory input may also be correlated with excitatory inputs projecting to the nearby dendritic hotspot (Figs. 5, 6), especially on the den- dritic tree of an excitatory neuron that is sensitive to changes in the external environment (Figs. 6, 8). Moreover, the model explains why the feature selectivity of these spines shows only a weak similarity, despite their correlations (Jia et al., 2010; Chen et al., 2011). When a synaptic cluster is carved by the heterosynaptic effect of common inhibitory inputs, and not by E-to-E interactions, the variability of feature selectivity within the cluster tends to be large, because inhibitory neurons typically have wider feature selectivity than excitatory neurons (Ma et al., 2010; Moore and Wehr, 2013). In addition, it should also be noted that, E-to-E heterosynaptic LTP is typically induced as a meta-plasticity over a timescale of minutes (Harvey and Svoboda, 2007), which by itself is insufficient to create a correlation-based synaptic cluster.

Second, the results in Figure 5 indicate that LTD at an excitatory synapse is offset by coincident inhibitory inputs to the nearby dendrite. Thus, LTD from low-frequency stimuli (Malenka and Bear, 2004) can be attenuated by coincident GABA uncaging near the stimulated spine. Note that this result would not contradict the previously reported GABA-driven heterosynaptic LTD by paired stimulation, because in that experiment, the excitatory spine was presumably too active to induce LTD in the absence of GABA (Hayama et al., 2013). Indeed, coincident GABAergic inputs may induce heterosynaptic LTD when combined with a moderately high-frequency presynaptic stimulation that in itself does not cause LTD (Blaise and Bronzino, 2003). The model also indicates that correlated inhibitory inputs are likely to suppress LTP at excitatory synapses if the heterosynaptic effect is sufficiently strong (Fig. 5G, blue area). This may be the case for spineprojecting inhibitory synapses.

The third implication of the model concerns the mechanism of binocular matching. Our model indicates that maturation of GABAergic inputs plays a critical role in binocular matching, and proposes a candidate mechanism for disruption of binocular matching by precocious GABA circuit maturation (B. S. Wang et al., 2013; Fig. 8). However, the phenomenon can also be explained by Hebbian plasticity plus some kind of meta-plasticity. If binocular matching is induced purely by Hebbian plasticity and not through a heterosynaptic mechanism, orientation selectivity after the matching should depend solely on the initial orientation selectivity of monocular inputs, assuming that the selectivity of presynaptic neurons remains the same. Especially when the contralateral input activity is larger than the ipsilateral input activity, the resultant orientation selectivity should approximately coincide with the original selectivity of the contralateral input. Alternatively, if the proposed mechanism is engaged during development, the refinement of orientation selectivity should also be influenced by the mean selectivity of the inhibitory input neurons. Thus, long-term imaging of monocular orientation selectivity of binocular neurons in V1 would reveal whether a covariance-based rule is sufficient to explain the phenomena, or whether some other mechanisms, including the one proposed here, also play a major role in the shift of orientation of selectivity.

\section{Carrier of heterosynaptic interaction}

Heterosynaptic plasticity has been observed over various spatial and temporal scales with different underlying molecular mechanisms (Nishiyama and Yasuda, 2015). In the case of heterosynaptic interactions involving milliseconds, single-atomic ions are strong candidates, as small molecules such as $\mathrm{IP}_{3}$ are too big to move rapidly from spine to spine (Santamaria et al., 2006). Under the assumption that changes in $\mathrm{Ca}^{2+}$ concentration at an unstimulated spine are crucial for heterosynaptic plasticity, $\mathrm{Ca}^{2+}$ influx/efflux from either intracellular or extracellular sources is 
necessary for induction of heterosynaptic plasticity. As inhibitory synaptic inputs often change the local $\mathrm{Ca}^{2+}$ concentration in the dendritic branch (Müllner et al., 2015), intracellular spreading of $\mathrm{Ca}^{2+}$ may be a major source of $\mathrm{Ca}^{2+}$ changes in nearby unstimulated spines. At the same time, because inhibitory inputs significantly modulate the membrane voltage of local dendrites (Gidon and Segev, 2012), a synaptic input should strongly drive $\mathrm{Ca}^{2+}$ influx/efflux through NMDA and VDCC from extracellular sources, even at nearby unstimulated spines. Additionally, most of the intracellular calcium ions are bound by calcium buffers (Higley and Sabatini, 2012), and the buffer concentration is also presumably important for induction of synaptic plasticity. In our model, both current-based interactions (Spine model) and calcium-based interactions (Reduced model) replicate the experimental results (Figs. 2 and 4, respectively). Nevertheless, our analytical result suggests that the heterosynaptic $\mathrm{Ca}^{2+}$ change typically needs to be comparable to the homosynaptic change to cause significant heterosynaptic plasticity through calcium-based interaction (Fig. $4 C, D$ ). Thus, this study highlights the possible importance of current-based interactions and a spine-specific influx/efflux of extracellular $\mathrm{Ca}^{2+}$ for inducing heterosynaptic plasticity.

Note that heterosynaptic interaction does not need to work on the order of milliseconds to influence the STDP time window. For example, E-to-E heterosynaptic LTD can be initiated by spreading of LTD-related molecules, not by messengers of neural activity (Hayama et al., 2013). Additionally, for a shift in the STDP time window, changes in the ratio of the calcium influx through NMDA and VDCC may play a crucial role (Paille et al., 2013).

\section{Inhibitory cell types}

Somatostatin-positive $\left(\mathrm{SOM}^{+}\right)$inhibitory neurons typically project to the apical dendrite, have a shorter membrane time constant than the typical timescale of calcium dynamics (Markram et al., 2004; Xu et al., 2013), and often show strong feature selectivity in comparison with other inhibitory neuron types (Ma et al., 2010). Thus, $\mathrm{SOM}^{+}$is the likely candidate for heterosynaptic STDP. However, our results do not exclude parvalbumin-positive $\left(\mathrm{PV}^{+}\right)$inhibitory neurons, which usually have projections to proximal dendrites, and are typically fast-spiking (Markram et al., 2004). In particular, h-STDP through $\mathrm{PV}^{+}$cells may play an important role in critical period plasticity (Takesian and Hensch, 2013).

\section{Related theoretical studies}

Previous theoretical studies show that excitatory heterosynaptic mechanisms such as dendritic spiking generate a functional synaptic clustering on the dendrite (Iannella and Tanaka, 2006; Legenstein and Maass, 2011; Kastellakis et al., 2016), and enriches computational capacity of the neuron (Poirazi and Mel, 2001; Legenstein and Maass, 2011). By contrast, we demonstrated in this study that an inhibitory synapse can induce clustering of nearby excitatory synapses (Figs. 5, 6). A characteristic of this inhibition-based clustering is the involvement of temporally precise activity to produce the detailed balance between local excitatory and inhibitory inputs. For instance, inhibition-based clustering is beneficial in the striatum, where temporally precise activity is crucial for motor coordination, and also in the hippocampus, where place cells exhibit temporally coordinated activity during spatial navigation. However, for modeling of contextual fear conditioning, excitation-based clustering is sufficient (Kastellakis et al., 2016), or potentially desirable, as temporally precise activity is not required for such a task.
For implementing the E/I balance at the soma, inhibitory STDP is a candidate underlying mechanism (Vogels et al., 2011; Kleberg et al., 2014). The proposed h-STDP model can be considered as an alternative explanation for the somatic detailed balance, because when all dendritic branches are balanced, the somatic membrane potential becomes naturally balanced. However, the model has further implications. First, unlike inhibitory STDP, in which inhibitory synapses passively counterbalance a pre-existing excitatory synaptic structure, in h-STDP the inhibitory synapses actively drive plasticity at nearby excitatory synapses (Fig. 5). Moreover, h-STDP enables a nonredundant synaptic weight organization on the dendrites by inducing the E/I balance locally at each dendritic branch (Fig. 6B). Previous synaptic plasticity models are unable to generate such dendritic synaptic weight distributions on their own, as the inhibitory STDP does not guarantee the balance at the dendrites, and the standard excitatory STDP does not support dendritic diversity (Fig. 6C). It is noted that, although h-STDP drives the local synaptic weights on a dendritic branch toward the detailed balance, the convergence to the exact balance is not guaranteed.

Recently, Yang and colleagues showed that an anti E/I balance on the dendritic tree can be beneficial for the gating of synaptic inputs, and suggested that heterosynaptic plasticity could be the underlying mechanism (Yang et al., 2016). In contrast, our model suggests that the anti-E/I balanced state is possible only under conditions of strong heterosynaptic inhibition, which is nonphysiological (Fig. 5G, blue area). This discrepancy may arise from the different definitions of presynaptic selectivity. In their work, the selectivity was defined based on the firing rate, whereas we used the spike correlation for defining the selectivity, as spike correlation presumably drives weight changes in STDP (Song et al., 2000).

Previous biophysical simulation studies reveal that synaptic plasticity at excitatory synapses critically depends on the inhibitory inputs at nearby dendrites (Cutsuridis, 2011; Bar-Ilan et al., 2013; Jedlicka et al., 2015; Wilmes et al., 2016), but these studies do not reveal much on the functional roles of the heterosynaptic plasticity. In particular, although the cancellation of plasticity by shunting inhibition was mentioned by Wilmes et al., (2016), their simple pairwise STDP model does not capture the differential effects of shunting inhibition on LTD and LTP depicted in our model (Fig. 5H-J). On the other hand, network modeling studies have found that heterosynaptic plasticity provides a homeostatic mechanism (Chen et al., 2013; Zenke et al., 2015), but in these models, heterosynaptic plasticity was modeled as a global homeostatic plasticity without any branch specificity, and its advantage over other homeostatic mechanisms was unclear. In this study, by considering intermediate abstraction with analytical but biologically plausible models, we proposed candidate mechanisms for experimental results that have not been modeled before, and revealed potential functions of h-STDP in neural circuit formation.

\section{Future work}

Although we fixed the weight of inhibitory synapses in our model to focus on the functions of h-STDP, inhibitory projections on excitatory neurons are known to show plasticity (Hennequin et al., 2017). In particular, a recent experimental study found that inhibitory and excitatory projections on the same postsynaptic cell show correlated weight changes, suggesting a heterosynaptic effect of inhibitory plasticity on excitatory plasticity (L. Wang and Maffei, 2014). This interaction among excitatory, inhibitory, 
and heterosynaptic plasticity should be studied in detail in the future.

In addition, an increasing number of recent studies indicates the importance of presynaptic changes for synaptic plasticity (Costa et al., 2015, 2017), suggesting the presence of active heterosynaptic plasticity at presynaptic axons. Correspondingly, previous experimental studies found vesicle superpools on axons that potentially regulate vesicle densities at neighboring boutons (Staras et al., 2010). On the other hand, computational studies on heterosynaptic plasticity including the present one are practically limited to changes in the postsynaptic dendrites. Hence, a theory on presynaptic heterosynaptic plasticity is awaited.

\section{References}

Bar-Ilan L, Gidon A, Segev I (2013) The role of dendritic inhibition in shaping the plasticity of excitatory synapses. Front Neural Circuits 6:118. CrossRef Medline

Bazelot M, Bocchio M, Kasugai Y, Fischer D, Dodson PD, Ferraguti F, Capogna M (2015) Hippocampal theta input to the amygdala shapes feedforward inhibition to gate heterosynaptic plasticity. Neuron 87: 1290-1303. CrossRef Medline

Bi GQ, Poo MM (1998) Synaptic modifications in cultured hippocampal neurons: dependence on spike timing, synaptic strength, and postsynaptic cell type. J Neurosci 18:10464-10472. Medline

Blaise JH, Bronzino JD (2003) Effects of stimulus frequency and age on bidirectional synaptic plasticity in the dentate gyrus of freely moving rats. Exp Neurol 182:497-506. CrossRef Medline

Branco T, Clark BA, Häusser M (2010) Dendritic discrimination of temporal input sequences in cortical neurons. Science 329:1671-1675. CrossRef Medline

Caporale N, Dan Y (2008) Spike timing-dependent plasticity: a Hebbian learning rule. Annu Rev Neurosci 31:25-46. CrossRef Medline

Chen JY, Lonjers P, Lee C, Chistiakova M, Volgushev M, Bazhenov M (2013) Heterosynaptic plasticity prevents runaway synaptic dynamics. J Neurosci 33:15915-15929. CrossRef Medline

Chen X, Leischner U, Rochefort NL, Nelken I, Konnerth A (2011) Functional mapping of single spines in cortical neurons in vivo. Nature 475: 501-505. CrossRef Medline

Chiu CQ, Lur G, Morse TM, Carnevale NT, Ellis-Davies GC, Higley MJ (2013) Compartmentalization of GABAergic inhibition by dendritic spines. Science 340:759-762. CrossRef Medline

Clopath C, Büsing L, Vasilaki E, Gerstner W (2010) Connectivity reflects coding: a model of voltage-based STDP with homeostasis. Nat Neurosci 13:344-352. CrossRef Medline

Costa RP, Froemke RC, Sjöström PJ, van Rossum MC (2015) Unified preand postsynaptic long-term plasticity enables reliable and flexible learning. eLife 4:e09457. CrossRef Medline

Costa RP, Mizusaki BE, Sjöström PJ, van Rossum MC (2017) Functional consequences of pre- and postsynaptic expression of synaptic plasticity. Philos Trans R Soc Lond B Biol Sci 372:20160153. CrossRef Medline

Cutsuridis V (2011) GABA inhibition modulates NMDA-R mediated spike timing dependent plasticity (STDP) in a biophysical model. Neural Netw 24:29-42. CrossRef Medline

Dorrn AL, Yuan K, Barker AJ, Schreiner CE, Froemke RC (2010) Developmental sensory experience balances cortical excitation and inhibition. Nature 465:932-936. CrossRef Medline

Espinosa JS, Stryker MP (2012) Development and plasticity of the primary visual cortex. Neuron 75:230-249. CrossRef Medline

Froemke RC (2015) Plasticity of cortical excitatory-inhibitory balance. Annu Rev Neurosci 38:195-219. CrossRef Medline

Gambino F, Pagès S, Kehayas V, Baptista D, Tatti R, Carleton A, Holtmaat A (2014) Sensory-evoked LTP driven by dendritic plateau potentials in vivo. Nature 515:116-119. CrossRef Medline

Gerstner W, Kempter R, van Hemmen JL, Wagner H (1996) A neuronal learning rule for sub-millisecond temporal coding. Nature 383:76-81. CrossRef Medline

Gidon A, Segev I (2012) Principles governing the operation of synaptic inhibition in dendrites. Neuron 75:330-341. CrossRef Medline

Graupner M, Brunel N (2007) STDP in a bistable synapse model based on CaMKII and associated signaling pathways. PLoS Comput Biol 3:e221. CrossRef Medline
Graupner M, Brunel N (2012) Calcium-based plasticity model explains sensitivity of synaptic changes to spike pattern, rate, and dendritic location. Proc Natl Acad Sci U S A 109:3991-3996. CrossRef Medline

Harvey CD, Svoboda K (2007) Locally dynamic synaptic learning rules in pyramidal neuron dendrites. Nature 450:1195-1200. CrossRef Medline

Hayama T, Noguchi J, Watanabe S, Takahashi N, Hayashi-Takagi A, EllisDavies GC, Matsuzaki M, Kasai H (2013) GABA promotes the competitive selection of dendritic spines by controlling local $\mathrm{Ca}^{2+}$ signaling. Nat Neurosci 16:1409-1416. CrossRef Medline

Hennequin G, Agnes EJ, Vogels TP (2017) Inhibitory plasticity: balance, control, and codependence. Annu Rev Neurosci 40:557-579. CrossRef Medline

Hensch TK (2005) Critical period plasticity in local cortical circuits. Nat Rev Neurosci 6:877-888. CrossRef Medline

Higley MJ, Sabatini BL (2012) Calcium signaling in dendritic spines. Cold Spring Harb Perspect Biol 4:a005686. CrossRef Medline

Hiratani N, Fukai T (2015) Mixed signal learning by spike correlation propagation in feedback inhibitory circuits. PLoS Comput Biol 11:e1004227. CrossRef Medline

Iannella N, Tanaka S (2006) Synaptic efficacy cluster formation across the dendrite via STDP. Neurosci Lett 403:24-29. CrossRef Medline

Jedlicka P, Benuskova L, Abraham WC (2015) A voltage-based STDP rule combined with fast BCM-like metaplasticity accounts for LTP and concurrent "heterosynaptic" LTD in the dentate gyrus in vivo. PLOS Comput Biol 11:e1004588. CrossRef Medline

Jia H, Rochefort NL, Chen X, Konnerth A (2010) Dendritic organization of sensory input to cortical neurons in vivo. Nature 464:1307-1312. CrossRef Medline

Kastellakis G, Silva AJ, Poirazi P (2016) Linking memories across time via neuronal and dendritic overlaps in model neurons with active dendrites. Cell Rep 17:1491-1504. CrossRef Medline

Kleberg FI, Fukai T, Gilson M (2014) Excitatory and inhibitory STDP jointly tune feedforward neural circuits to selectively propagate correlated spiking activity. Front Comput Neurosci 8:53. CrossRef Medline

Kleindienst T, Winnubst J, Roth-Alpermann C, Bonhoeffer T, Lohmann C (2011) Activity-dependent clustering of functional synaptic inputs on developing hippocampal dendrites. Neuron 72:1012-1024. CrossRef Medline

Koch C (1998) Biophysics of computation: information processing in single neurons. New York: Oxford UP.

Kuhlman SJ, Tring E, Trachtenberg JT (2011) Fast-spiking interneurons have an initial orientation bias that is lost with vision. Nat Neurosci 14: 1121-1123. CrossRef Medline

Legenstein R, Maass W (2011) Branch-specific plasticity enables selforganization of nonlinear computation in single neurons. J Neurosci 31: 10787-10802. CrossRef Medline

Letzkus JJ, Kampa BM, Stuart GJ (2006) Learning rules for spike timingdependent plasticity depend on dendritic synapse location. J Neurosci 26:10420-10429. CrossRef Medline

Liu G (2004) Local structural balance and functional interaction of excitatory and inhibitory synapses in hippocampal dendrites. Nat Neurosci 7:373-379. CrossRef Medline

London M, Häusser M (2005) Dendritic computation. Annu Rev Neurosci 28:503-532. CrossRef Medline

Lüscher C, Malenka RC (2012) NMDA receptor-dependent long-term potentiation and long-term depression (LTP/LTD). Cold Spring Harb Perspect Biol 4:a005710. CrossRef Medline

Ma WP, Liu BH, Li YT, Huang ZJ, Zhang LI, Tao HW (2010) Visual representations by cortical somatostatin inhibitory neurons-selective but with weak and delayed responses. J Neurosci 30:14371-14379. CrossRef Medline

Malenka RC, Bear MF (2004) LTP and LTD: an embarrassment of riches. Neuron 44:5-21. CrossRef Medline

Mallet N, Moine C, Charpier S, Gonon F (2005) Feedforward inhibition of projection neurons by fast-spiking GABA interneurons in the rat striatum in vivo. J Neurosci 25:3857-3869. CrossRef Medline

Markram H, Toledo-Rodriguez M, Wang Y, Gupta A, Silberberg G, Wu C (2004) Interneurons of the neocortical inhibitory system. Nat Rev Neurosci 5:793-807. CrossRef Medline

Marlin JJ, Carter AG (2014) GABA-A receptor inhibition of local calcium signaling in spines and dendrites. J Neurosci 34:15898-15911. CrossRef Medline 
Mel BW, Schiller J (2004) On the fight between excitation and inhibition: location is everything. Sci STKE 2004:PE44. CrossRef Medline

Moore AK, Wehr M (2013) Parvalbumin-expressing inhibitory interneurons in auditory cortex are well-tuned for frequency. J Neurosci 33: 13713-13723. CrossRef Medline

Müllner FE, Wierenga CJ, Bonhoeffer T (2015) Precision of inhibition: dendritic inhibition by individual GABAergic synapses on hippocampal pyramidal cells is confined in space and time. Neuron 87:576-589. CrossRef Medline

Nishiyama J, Yasuda R (2015) Biochemical computation for spine structural plasticity. Neuron 87:63-75. CrossRef Medline

Oh WC, Parajuli LK, Zito K (2015) Heterosynaptic structural plasticity on local dendritic segments of hippocampal CA1 neurons. Cell Rep 10:162169. CrossRef Medline

Paille V, Fino E, Du K, Morera-Herreras T, Perez S, Kotaleski JH, Venance L (2013) GABAergic circuits control spike-timing-dependent plasticity. J Neurosci 33:9353-9363. CrossRef Medline

Petersen CC, Malenka RC, Nicoll RA, Hopfield JJ (1998) All-or-none potentiation at CA3-CA1 synapses. Proc Natl Acad Sci U S A 95:4732-4737. CrossRef Medline

Poirazi P, Mel BW (2001) Impact of active dendrites and structural plasticity on the memory capacity of neural tissue. Neuron 29:779-796. CrossRef Medline

Poirazi P, Brannon T, Mel BW (2003) Pyramidal neuron as two-layer neural network. Neuron 37:989-999. CrossRef Medline

Santamaria F, Wils S, De Schutter E, Augustine GJ (2006) Anomalous diffusion in Purkinje cell dendrites caused by spines. Neuron 52:635-648. CrossRef Medline

Shouval HZ, Bear MF, Cooper LN (2002) A unified model of NMDA receptor-dependent bidirectional synaptic plasticity. Proc Natl Acad Sci U S A 99:10831-10836. CrossRef Medline

Sjöström PJ, Häusser M (2006) A cooperative switch determines the sign of synaptic plasticity in distal dendrites of neocortical pyramidal neurons. Neuron 51:227-238. CrossRef Medline

Sjöström PJ, Turrigiano GG, Nelson SB (2001) Rate, timing, and cooperativity jointly determine cortical synaptic plasticity. Neuron 32:11491164. CrossRef Medline

Smith SL, Smith IT, Branco T, Häusser M (2013) Dendritic spikes enhance stimulus selectivity in cortical neurons in vivo. Nature 503:115-120. CrossRef Medline

Song S, Miller KD, Abbott LF (2000) Competitive Hebbian learning through spike-timing-dependent synaptic plasticity. Nat Neurosci 3:919926. CrossRef Medline

Staras K, Branco T, Burden JJ, Pozo K, Darcy K, Marra V, Ratnayaka A, Goda $\mathrm{Y}$ (2010) A vesicle superpool spans multiple presynaptic terminals in hippocampal neurons. Neuron 66:37-44. CrossRef Medline

Takahashi N, Kitamura K, Matsuo N, Mayford M, Kano M, Matsuki N, Ikegaya Y (2012) Locally synchronized synaptic inputs. Science 335: 353-356. CrossRef Medline

Takesian AE, Hensch TK (2013) Balancing plasticity/stability across brain development. Prog Brain Res 207:3-34. CrossRef Medline

Tsukada M, Aihara T, Kobayashi Y, Shimazaki H (2005) Spatial analysis of spike-timing-dependent LTP and LTD in the CA1 area of hippocampal slices using optical imaging. Hippocampus 15:104-109. CrossRef Medline

Vogels TP, Sprekeler H, Zenke F, Clopath C, Gerstner W (2011) Inhibitory plasticity balances excitation and inhibition in sensory pathways and memory networks. Science 334:1569-1573. CrossRef Medline

Wang BS, Sarnaik R, Cang J (2010) Critical period plasticity matches binocular orientation preference in the visual cortex. Neuron 65:246-256. CrossRef Medline

Wang BS, Feng L, Liu M, Liu X, Cang J (2013) Environmental enrichment rescues binocular matching of orientation preference in mice that have a precocious critical period. Neuron 80:198-209. CrossRef Medline

Wang L, Maffei A (2014) Inhibitory plasticity dictates the sign of plasticity at excitatory synapses. J Neurosci 34:1083-1093. CrossRef Medline

Wilmes KA, Sprekeler H, Schreiber S (2016) Inhibition as a binary switch for excitatory plasticity in pyramidal neurons. PLoS Comput Biol 12: e1004768. CrossRef Medline

Wilson NR, Ty MT, Ingber DE, Sur M, Liu G (2007) Synaptic reorganization in scaled networks of controlled size. J Neurosci 27:13581-13589. CrossRef Medline

Xu H, Jeong HY, Tremblay R, Rudy B (2013) Neocortical somatostatinexpressing GABAergic interneurons disinhibit the thalamorecipient layer 4. Neuron 77:155-167. CrossRef Medline

Yang GR, Murray JD, Wang XJ (2016) A dendritic disinhibitory circuit mechanism for pathway-specific gating. Nat Commun 7:12815. CrossRef Medline

Yazaki-Sugiyama Y, Kang S, Câteau H, Fukai T, Hensch TK (2009) Bidirectional plasticity in fast-spiking GABA circuits by visual experience. Nature 462:218-221. CrossRef Medline

Zenke F, Agnes EJ, Gerstner W (2015) Diverse synaptic plasticity mechanisms orchestrated to form and retrieve memories in spiking neural networks. Nat Commun 6:6922. CrossRef Medline 